\title{
Anleitungen rezipieren, Anleitungen produzieren: Empirische Befunde zu kognitiven Prozessen bei Übersetzungsnovizen und Übersetzungsprofis
}

\begin{abstract}
The article presents results from the research project TransComp, a process-oriented longitudinal study which investigates the development of translation competence in 12 students of translation over a period of three years and compares it with that of 10 professional translators. The article focuses on the translation products and processes of six novices and five professional translators who had to translate operating instructions from English (their L2) into German (their L1). During the translation process, the participants had to think aloud. They were allowed to use any external reference resources they wished. Their think-aloud and all their activities on the computer were screen-recorded using CamtasiaStudio. In addition, their writing processes were logged using the keystroke-logging software Translog. Immediately after the experiments, they were asked to complete a questionnaire. The data gained in this multi-method approach are analyzed both from a product-oriented and from a process-oriented perspective. For the product-oriented analyses, an error classification was developed that combines a linguistic characterization of the mistakes with a functional approach of determining how detrimental these are with regard to the target text function. The process-oriented analyses are based on the subjects' think-aloud, their activities recorded with CamtasiaStudio, their questionnaires and their log files. These process data are analyzed to determine the subjects' problem awareness and their problem-solving competence. Based on the findings, implications for translation pedagogy and text production didactics are discussed.
\end{abstract}

\section{Kontext der Studie und Versuchspersonen}

Bei den hier präsentierten Ergebnissen handelt es sich um Teilbefunde aus der Longitudinalstudie TransComp ${ }^{1}$, in der die Entwicklung der translatorischen Kompetenz von zwölf Studierenden im Bachelor-Übersetzungsstudiengang „Transkulturelle Kommunikation“ am Institut für Theoretische und Angewandte Translationswissenschaft der Karl-Franzens-Universität Graz von Studienbeginn an über einen Zeitraum von drei Jahren untersucht und mit der translatorischen Kompetenz von zehn professionellen Übersetzern verglichen wird (Näheres in Göpferich 2009a). Eines der Ziele der Longitudinalstudie besteht darin, Aufschluss über die verschiedenen Teilkompetenzen zu erhalten, die translatorische Kompetenz insgesamt ausmachen, aber auch darüber, in welcher Weise diese Teilkompetenzen in verschiedenen Ausbildungs- bzw. Kompetenzstadien (angehender) Übersetzer interagieren und wie die Entwicklung dieser Teilkompetenzen und ihre Interaktion gefördert werden können (s. zur Modellierung translatorischer Kompetenz Göpferich 2008: 143 ff.).

Während im Projekt TransComp an der Universität Graz und im Projekt CTP(Capturing Translation Processes) an der Zürcher Hochschule für Angewandte Wissenschaften (Ehrensberger-Dow

1 TransComp wird als Projekt Nummer P20908-G03 vom Fonds zur Förderung der wissenschaftlichen Forschung (FWF), Österreichs zentraler Einrichtung zur Förderung der Grundlagenforschung, von September 2008 bis August 2011 gefördert.

\footnotetext{
* Susanne Göpferich

Institut für Theoretische und Angewandte Translationswissenschaft (ITAT)

Karl-Franzens-Universität Graz

Merangasse 70

A-8010 Graz

susanne.goepferich@uni-graz.at
} 
2009) die Entwicklung translatorischer Kompetenz erstmals in ihrer Kontinuität an denselben Versuchspersonen über einen mehrjährigen Zeitraum untersucht wird, liegen bereits zahlreiche Befunde vor, die in kontrastiven Studien gewonnen wurden, in denen zu nur einem Zeitpunkt zwei oder drei Gruppen von Versuchspersonen mit unterschiedlichem Kompetenzgrad miteinander verglichen wurden. Die Befunde aus diesen Studien können hier aus Platzgründen nicht zusammengefasst werden, finden sich jedoch in einem Überblick in Göpferich (2008: 168 ff.) und werden hier in die Diskussion der Ergebnisse einbezogen. Erwähnenswert ist darüber hinaus die Dissertation von Künzli (2003) als eine der größer angelegten Studien, in der die Übersetzungsprodukte und -prozesse von zehn deutschsprachigen Versuchspersonen aus der Schweiz (fünf Studierenden und fünf professionellen Übersetzern) sowie zehn Schweden (vier Studierenden und sechs professionellen Übersetzern) untersucht wurden, die jeweils auch einen Anleitungstext übersetzen und dabei laut denken mussten, in Künzlis Studie allerdings aus dem Französischen ins Deutsche bzw. Schwedische. Obwohl auch Künzli u. a. an Übersetzungsstrategien interessiert war, muss betont werden, dass sein Strategiebegriff, den er von Chesterman (1997) übernimmt und mit dem eher translatorische Shifts gemeint sind, die sich an der sprachlichen Oberfläche des Produkts zeigen, ein völlig anderer ist, als der prozessorientierte, der der vorliegenden Studie zugrunde liegt (s. hierzu Abschnitt 4.2), weshalb hier kein näherer Vergleich der Ergebnisse angestellt werden soll.

Den Ausgangspunkt der vorliegenden Teilstudie bilden die Übersetzungsprodukte und -prozesse aus insgesamt 11 Versuchen, in denen jeweils $50 \%$ der studentischen Versuchspersonen (Vpn), die an TransComp teilnahmen, und 50 \% der professionellen Vpn den Bedienungsanleitungstext übersetzten, der in Anhang A zusammen mit dem Übersetzungsauftrag abgedruckt ist, der den Vpn mit ausgehändigt wurde. Die Studierenden befanden sich zum Übersetzungszeitpunkt am Anfang ihres ersten Semesters. Es handelte sich dabei um fünf Studentinnen und einen Studenten im Alter von 19 bis 22 Jahren. Fünf davon sind österreichische Staatsbürger, eine ist Deutsche. Ihre Muttersprache ist ausnahmslos Deutsch, ihre 1. Fremdsprache in der Schule und im Übersetzungsstudium Englisch, wobei ihre letzten Schulnoten in Deutsch und Englisch bis auf eine Ausnahme „sehr gut“ oder ,gut“ waren. Die Ausnahme ist eine Studentin, bei der die letzte Schulnote in Englisch nur „,befriedigend“ war. Alle nahmen freiwillig an der Studie teil.

Die fünf professionellen Vpn haben alle eine universitäre Translationsausbildung abgeschlossen; zu ihren Arbeitssprachen gehören Deutsch und Englisch, und sie verfügen über mindestens zehn Jahre Berufserfahrung (zwischen 11 und 40 Jahre) im Bereich Übersetzen/Dolmetschen.

\section{Datenerhebungsmethoden}

Die Vpn übersetzten den Text in Anhang A im Editor des Schreib-Logging-Programms Translog 2006, das alle Tastenanschläge und Mausklicks sowie die Zeitintervalle dazwischen aufzeichnet (Jakobsen 1999). Ihnen standen gedruckte ein- und zweisprachige Wörterbücher zur Verfügung, die sie ihren Wünschen entsprechend um weitere gedruckte und elektronische Wörterbücher ergänzen durften. Außerdem standen ihnen alle Recherche-Möglichkeiten im Internet zur Verfügung. Während des Übersetzens mussten die Vpn laut denken, worin sie zum Zeitpunkt des Versuchs in einer Aufwärmphase und zwei vorausgehenden Versuchen schon einige Erfahrung gesammelt hatten. Ihr lautes Denken sowie alle Aktionen am Bildschirm wurden mit der ScreenRecording-Software Camtasia Studio aufgezeichnet. Die Nutzung gedruckter Nachschlagewerke protokollierte die Versuchsleiterin im Hintergrund. Das laute Denken der Vpn und ihre Aktivitäten am Bildschirm sowie ihre Recherchen in den gedruckten Wörterbüchern wurden in XMLbasierten Transkripten dokumentiert, die hier als Übersetzungsprotokolle bezeichnet werden (s. zu den Transkriptionskonventionen Göpferich 2010). Unmittelbar nach Abschluss jedes Übersetzungsversuchs füllten die Vpn einen Fragebogen zu ihrem Befinden während des Versuchs und ihren Übersetzungsproblemen aus, die sie auflisten und kommentieren sollten. Außerdem war in den Fragebögen u. a. auch eine Einschätzung des Schwierigkeitsgrades des Textes abzugeben. 


\section{Datendokumentation und Problemstellenbestimmung}

Die Daten (Übersetzungsprotokolle, Camtasia-Aufzeichnungen, Log-Dateien, Zieltexte mit Fehlerbewertung) sowie die Ausgangstexte (AT) mit Übersetzungsauftrag stehen in einem Asset-Management-System (AMS) im Internet zur Verfügung, in dem die Übersetzungsprotokolle auch parallel zu den Camtasia-Aufzeichnungen angeschaut werden können (s. Göpferich/Bayer-Hohenwarter/Stigler 2008 ff.). Bis Juli 2011, wenn die letzte Versuchsreihe durchgeführt wird, sind diese Daten jedoch passwortgeschützt, um sicherzustellen, dass die Versuchspersonen vorab keinen Zugang zu Material haben, mit dem sie erst in zukünftigen Versuchen konfrontiert werden.

In den Übersetzungsprotokollen wurden als Interpretationskategorie auch die Problemstellen der Vpn ausgezeichnet. Zu ihrer Identifikation wurde eine adaptierte Version von Krings' (1986: 121) Problemstellenindikatoren verwendet, die eine konsistente und intersubjektiv einheitliche Ermittlung von Übersetzungsproblemen ermöglichen sollen. Krings unterscheidet zwischen „primären Problemindikatoren“, zu denen er solche zählt, die zweifelsfrei auf das Vorliegen einer Problemstelle schließen lassen, und „sekundären Problemindikatoren“, die auf eine Problemstelle hindeuten können, dies aber nicht zweifelsfrei tun. Aus diesem Grund wertet Krings nur solche Phänomene als Übersetzungsprobleme, auf die mindestens ein primärer Indikator oder aber mindestens zwei sekundäre Indikatoren hinweisen.

Wie in Krings' Studie wurden auch in der vorliegenden die folgenden Phänomene als primäre Problemindikatoren gewertet: 1 . die explizite oder implizite Problemidentifikation durch den Übersetzer, z. B. in einer Äußerung wie „da weiß ich jetzt nicht, wie ich das übersetzen soll“, 2. jede Hilfsmittelbenutzung und 3. Lücken im zielsprachlichen Text. Krings' Liste sekundärer Problemindikatoren musste für die vorliegende Studie modifiziert werden. Lediglich die folgenden fünf Phänomene aus Krings' Liste wurden als sekundäre Problemindikatoren gewertet: 1 . konkurrierende tentative Übersetzungsäquivalente, 2. die negative Bewertung einer Zieltexteinheit durch den Übersetzer, 3. ungefüllte Pausen von mehr als 3 Sekunden Dauer, 4. bestimmte paralinguale Indikatoren, wie Stöhnen und Seufzen, und 5. das Fehlen einer primären Äquivalentassoziation. Transkriptstellen wurden wohlgemerkt nur dann als Problemstelle gewertet, wenn mindestens zwei der sekundären Indikatoren darauf hinwiesen. Zu dieser Regel gibt es eine Ausnahme: Wenn eine Versuchsperson zu einem späteren Zeitpunkt ein Übersetzungsproblem, das schon anhand der Indikatoren zuvor als solches identifiziert worden war, wieder aufnimmt, so reicht ein einziger sekundärer Indikator, um diese Wiederaufnahme als Problemstelle zu werten. Transkriptstellen, an denen zwar hinreichend Indikatoren für eine Problemstelle vorlagen, aber unklar blieb, was das Problem ausgelöst haben mag, wurden nicht als Problemstellen gewertet. Kommentare zur Schwierigkeit des Textes oder dazu, ob der Vpn der Text lag, wurden ebenfalls nicht als Problemstellen gewertet. Übersetzungsprobleme in diesem Sinne können in allen Phasen des Übersetzungsprozesses auftreten, auch in der Orientierungs- und der Revisionsphase. Der Start-Tag für eine Problemstelle im Transkript wurde unmittelbar vor der Äußerung bzw. Aktion gesetzt, die auf das Problem hindeutet, der Ende-Tag an der Stelle, an der die Problemlösung abgeschlossen wurde oder sich die Vpn anderen Aspekten zuwendet (eine nähere Diskussion von Krings' sekundären Problemindikatoren findet sich in Göpferich 2010).

\section{Datenanalyse}

Bei der Datenanalyse wurden ein produktorientierter und ein prozessorientierter Ansatz verfolgt.

\subsection{Produktorientierter Ansatz}

Der produktorientierte Ansatz besteht darin, dass zunächst der Zieltext (ZT) jeder Vpn analysiert und bewertet wurde. Dabei kam das Fehlerklassifikationsschema in Anhang B zum Einsatz. Alle Fehler im ZT wurden, wie aus der Tabelle ersichtlich, zunächst nach primär linguistischen Kriterien in formale Fehler, Wortwahlfehler, Grammatikfehler, Fehler auf der Textebene und sonstige Fehler (d. h. Fehler im Bereich der Idiomatik und der Textsortenkonventionen und Fehler im Be- 
reich Kulturspezifik) mit den in der Tabelle in Anhang B ausgewiesenen Unterkategorien eingeteilt. Die Gewichtung dieser Fehler erfolgte auf einer dreistufigen Skala (-0,5 / -1 / -1,5) nach dem Grad, in dem die kommunikative Funktion des ZT gestört wird. Ein Rechtschreibfehler, der nicht sinnentstellend ist, wurde also als halber Fehler $(-0,5)$ gewertet, die Einfügung einer Negation an einer Stelle, an der eine affirmative Aussage gemacht werden muss und die Negation zu einer Gefährdung des Benutzers führen kann, als anderthalber Fehler (-1,5). Die Fehlerbewertung wurde jeweils von drei Personen mit einem abgeschlossenen universitären Übersetzungsstudium durchgeführt. Stellen, an denen es zu Diskrepanzen in der Fehlerbewertung zwischen ihnen kam, wurden gemeinsam diskutiert, bis ein Konsens erreicht wurde. Bei den Fehlern wurde außerdem unterschieden, ob es sich um Fehler handelt, die auf Mängel in der muttersprachlichen Kompetenz (MuKo) zurückzuführen sind und auch übersetzungsunabhängig aufträten und ggf. behoben werden könnten, und solchen, die translationsspezifisch sind (TransKo). Alle Zweifelsfälle, insbesondere auch interferenzbedingte Fehler, wurden den translationsspezifischen Fehlern zugerechnet (s. die Tabellen in Göpferich 2009b).

\subsection{Prozessorientierter Ansatz}

Für die Prozessanalysen wurden die Übersetzungsprotokolle und die Ergebnisse aus den versuchsbegleitenden Fragebögen herangezogen, in denen die Vpn unmittelbar nach Abschluss des Übersetzungsversuches u. a. die Problemstellen auflisten und kommentieren sollten, die sie während des Übersetzens selbst als solche empfunden hatten, und eine Einschätzung des Schwierigkeitsgrades des Textes abzugeben hatten. ${ }^{2}$

Zunächst wurden die Versuchsdauer sowie die Dauer der verschiedenen Übersetzungsphasen (Orientierungsphase - pre-phase; Übersetzungsphase - main-phase; Revisionsphase(n) - postphase bzw. post-phase 1, post-phase 2 etc.) erhoben, die in den Übersetzungsprotokollen dokumentiert sind, sowie die Übersetzungsgeschwindigkeit in Wörtern pro Minute ermittelt (s. die Rubrik „Translation time and translation speed“ in den Tabellen in Göpferich 2009b).

Auf der Basis der Übersetzungsprotokolle und der Angaben der Vpn in den versuchsbegleitenden Fragebögen wurde außerdem ermittelt, inwiefern die Stellen, an denen nach den Ergebnissen der produktorientierten Analyse Fehler auftraten, von den Vpn reflektiert wurden. Als reflektiert wurde eine Fehlerstelle dann gewertet, wenn die Vpn zu ihr im Übersetzungsprotokoll zumindest eine Alternative in Erwägung gezogen oder ihre Lösung selbst negativ kommentiert hat oder aber eine Textstelle bewusst nicht übersetzt hat, die eine Übersetzung erfordert hätte. In den Tabellen in Göpferich (2009b) sind die reflektierten Fehlerstellen in der Rubrik „Errors“ als Werte in Klammern hinter den Fehlerzahlen angegeben.

Zusätzlich wurden die anhand der in Abschnitt 3 erwähnten Indikatoren identifizierten Probleme der Vpn klassifiziert in Verstehensprobleme, Wiedergabeprobleme und kombinierte Verstehens- und Wiedergabeprobleme, wobei zur letzteren Kategorie auch alle Probleme gerechnet wurden, bei denen nicht klar zu entscheiden war, ob es sich eher um ein Verstehens- oder ein Wiedergabeproblem handelt. Die Klassifikation der Probleme erfolgte auf der Basis der Übersetzungsprotokolle und der Angaben der Vpn in den versuchsbegleitenden Fragebögen. Diese drei genannten Kategorien von Problemen wurden nochmals in gelöste und ungelöste unterteilt. Ausschlaggebend für die Beurteilung, ob ein Problem als gelöst oder ungelöst zu betrachten ist, war nicht die Perspektive der Vpn, sondern die Frage, ob das Übersetzungsprodukt an der betreffenden Stelle korrekt ist oder nicht. Bei kombinierten Verstehens- und Wiedergabeproblemen können sich die Attribute „gelöst“ und „ungelöst“ folglich nur auf die korrekte bzw. falsche Wiedergabe im ZT beziehen, d. h., ein kombiniertes Verstehens- und Wiedergabeproblem gilt auch dann als ungelöst, wenn der Verstehensprozess zwar erfolgreich verlaufen sein mag, aber dennoch

2 Eine weitere Datenquelle sind die Translog-Dateien, auf die für die vorliegende Teilstudie aber nur in Zweifelsfällen zurückgegriffen wurde, z. B. um festzustellen, ob eine Vpn etwas nur vor sich her sagte oder es sich auch diktierte oder ob eine Vpn Zwischenversionen produzierte, ohne sie zu verbalisieren, was in den Log-Dateien abzulesen ist. 
nicht zu einer korrekten Wiedergabe führte (s. hierzu die Rubrik „Problem incidents and types“ in den Tabellen in Göpferich 2009b)

Als zentraler Bestandteil der Analysen wurde außerdem analysiert, inwiefern die Vpn strategisch vorgingen. Unter einer strategischen Vorgehensweise wird hier ein Problemlösungsverhalten verstanden, bei dem sich die Vpn der Kriterien bewusst ist oder sich (systematisch) die Kriterien erarbeitet, die eine ZT-Einheit erfüllen muss, um als adäquate Übersetzung eines AT-Segments betrachtet werden zu können. Eine strategische Vorgehensweise kann damit als Gegenstück zu reinem Raten oder Sich-auf-sein-Gefühl-Verlassen verstanden werden, zu dem Studierende häufig Zuflucht nehmen, wenn ihnen die relevanten Kriterien nicht bewusst sind. Das Ausmaß der Strategiegeleitetheit der Entscheidungen einer Vpn wird hier als ein Indikator für strategische Kompetenz betrachtet (s. hierzu das Translationskompetenzmodell in Göpferich 2008: 155 ff.). Dieser Indikator muss jedoch jeweils vor dem Hintergrund der Angemessenheit der Übersetzungslösungen betrachtet werden. Daher werden hier prozessorientierte Analysen mit produktorientierten kombiniert, die der Bestimmung der Übersetzungsqualität dienen. Um Aufschluss über die Wege zu erhalten, auf denen Übersetzungsentscheidungen zustande kommen, wurden für ausgewählte Problemstellen die Problemlösungspfade der Vpn untersucht, also alle Maßnahmen, die ergriffen wurden, und Überlegungen, die angestellt wurden, um die entsprechenden Probleme zu lösen. Drei solcher Problemstellen unterschiedlicher Art werden in Abschnitt 5.2 exemplarisch behandelt.

Vor dem Hintergrund, dass zahlreiche Anleitungen eine mangelhafte Qualität aufweisen und man von einem idealen Übersetzer (im Folgenden Übersetzungsexperte genannt) erwartet, dass er Schwachstellen nicht in die zielsprachliche Version übernimmt, wurde außerdem noch eine Aufstellung der allgemeinen Überlegungen gemacht, die ein Übersetzungsexperte angesichts des AT anstellen sollte, um eine optimale ZT-Qualität erreichen zu können. Für jede dieser Überlegungen wurde anhand der Übersetzungsprotokolle überprüft, ob die Vpn diese Überlegung angestellt haben. Diese Überlegungen sind im Folgenden aufgelistet. Welche von ihnen von den einzelnen Vpn angestellt wurden, geht ebenfalls aus den Tabellen 1 und 2 in Göpferich (2009b) hervor (s. Rubrik „Reflections“).

1. Ein Übersetzungsexperte verlangt nach dem Gerät, um unklar dargestellte Sachverhalte ausprobieren zu können. Abbildungen sind hier eine Hilfe, reichen aber im vorliegenden Text nicht zur Klärung aller Unklarheiten aus.

2. Von einem Übersetzungsexperten kann man erwarten, dass er sich für die konsistente Verwendung eines Illokutionsindikators für Instruktionen entscheidet (Imperativ oder imperativischer Infinitiv). Grundsätzlich sind beide möglich; da es sich jedoch bei der Mixeranleitung um eine Anleitung für Laien handelt, entspricht die Verwendung des Imperativs im Deutschen eher den Konventionen.

3. Im Zusammenhang mit Punkt 1 ist von einem Experten zu erwarten, dass er die mögliche Existenz eines unternehmensspezifischen Redaktionshandbuchs (Style Guides) thematisiert und terminologische Konsistenz anstrebt. In den Tabellen 1 und 2 in Göpferich (2009b) wurde ein ,ja“ vergeben, wenn zumindest einer der beiden Aspekte im Übersetzungsprotokoll thematisiert wurde.

4. Unter Punkt 5 weist der Ausgangstext eine missverständliche Formulierung auf (,while you are using it“). Gemeint ist mit dieser Formulierung während man das Gerät zwar einsatzbereit hat, aber gerade nicht damit mixt. Von einem Übersetzungsexperten kann man hier erwarten, dass er diese Stelle im ZT optimiert (siehe hierzu ausführlich Abschnitt 5.2.2).

5. Im AT werden Bedingungen, deren Vorliegen aus Sicherheitsgründen unbedingtherbeizuführen ist, nicht explizit durch direktive Sprechakte als solche charakterisiert. Dies gilt für die folgende Formulierung: "With the control switch (D) on 0, the attachments can be removed simply by pressing the eject button.” Aus produkthaftungsrechtlicher Sicht dürfen sich die 
Rührwerkzeuge durch Drücken der Auswurftastenur lösen lassen, wenn das Gerät ausgeschaltet ist. Bei manchen Mixern geht das aber auch bei laufendem Gerät, was die Rührwerkzeuge zu gefährlichen Geschossen werden lassen und daher rechtlich schwerwiegende Folgen für den Hersteller haben kann. Wenn es schon möglich ist, die Einsätze auch bei laufendem Gerät zu lösen, so muss davor eindringlich in einem Sicherheitshinweis gewarnt werden. Dies kann geschehen, indem man darauf hinweist, dass der Stufenschalter vor dem Betätigen der Auswurftaste unbedingt auf 0 stehen muss. Auch im Englischen wäre es also sicherer, die obige Formulierung, eingebettet in einen Sicherheitshinweis, wie folgt zu paraphrasieren: "Check that the control switch is in position 0. To release the attachments, press the eject button.” Zumindest weist ein Übersetzungsexperte seinen Auftraggeber auf die Notwendigkeit entsprechender Sicherheitshinweise hin.

6. Im AT kommen Begriffe vor, für die es in den verschiedenen deutschsprachigen Ländern Benennungsunterschiede gibt. Dieses Problem muss reflektiert werden. In der Regel wird aus ökonomischen Gründen nur eine deutsche Version erstellt. In dieser sollten länderneutrale Benennungen verwendet werden; wo dies nicht möglich ist, ist aufgrund der größten Sprecherzahl dem Bundesdeutschen der Vorzug zu geben (s. hierzu ausführlich Abschnitt 5.2.3).

Während die o. g. Punkte von einem Übersetzungsexperten unbedingt beachtet werden müssen, damit eine akzeptable Übersetzung entsteht, handelt es sich bei den nachfolgenden Punkten um solche, deren Reflexion und/oder Beachtung von extremer sprachlicher Sensibilität zeugen, insbesondere was die typographische und sprachliche Gestaltung von Anleitungen betrifft. Ihre Nichtbeachtung führt nicht automatisch zu einem nicht akzeptablen Übersetzungsprodukt:

7. Punkt 5 der Anleitung weist eine eigene fettgedruckte Überschrift auf; eine solche wäre aus Gründen der Konsistenz auch bei den beiden vorausgehenden Punkten wünschenswert (z. B. Insertion of attachments und Positioning the mixer).

8. Die Ziffern für die Geschwindigkeitsstufen unter „Recommendations“ sollten jeweils bei Ersterwähnung der Geschwindigkeitsstufe erscheinen, was bei „medium speed“ und „fast speed“ jedoch erst bei ihrer Zweiterwähnung der Fall ist.

9. Der boost button erscheint im Gegensatz zu allen anderen Schaltern in Fettdruck. Hier ist Einheitlichkeit herzustellen. Dies kann jedoch in den Übersetzungen der Vpn nicht überprüft werden, da die Formatierung aufgrund des Einsatzes von Translog mit seinen beschränkten Editierfunktionen nicht Bestandteil des Übersetzungsauftrags war.

10. Die Beschreibung der Geschwindigkeitsstufen erfolgt jeweils in vollständigen Sätzen mit „,is used to"; die Beschreibung des Turboschalters ist jedoch direkt nach einem Doppelpunkt, dem fälschlicherweise ein Leerzeichen vorausgeht, elliptisch angeschlossen. Hier ist auf Parallelität der Konstruktionen zu achten.

Eines der Ziele, das mit den oben beschriebenen Analysen verfolgt wurde, ist festzustellen, ob die Vpn für Übersetzungsprobleme sensibilisiert sind. Denn Problemsensibilität stellt eine wesentliche Voraussetzung für optimale Übersetzungsentscheidungen dar.

\section{Ergebnisse}

\section{1. Übersetzungsdauer, -phasen, Produktqualität und Übersetzungsprobleme}

Für die Übersetzung der Anleitung benötigten die Studierenden mit durchschnittlich 72,3 Minuten ca. 1,6-mal so viel Zeit wie die professionellen Übersetzer mit im Schnitt 45,4 Minuten. Schafften die professionellen Übersetzer 5,3 Wörter pro Minute, so waren es bei den Studierenden nur 3,3. Trotz des wesentlich höheren Zeitbedarfs machten die Studierenden durchschnittlich ein Drittel mehr Fehler $(18,9)$ als die professionellen Übersetzer $(12,4)$. Bei den Studierenden lie- 
gen 12,2 \% aller Fehler in mangelnder muttersprachlicher Kompetenz begründet, bei den professionellen Übersetzern nur 8,1 \%. Die restlichen Fehler liegen in der translatorischen Kompetenz begründet oder könnten zumindest auf diese zurückzuführen sein (s. hierzu Abschnitt 4.1). Die gravierendsten Defizite weisen beide Gruppen im Bereich der Wortwahlfehler auf, von denen die meisten semantische Fehler sind. Die Wortwahlfehler machen bei den Studierenden durchschnittlich 14,5 Fehler (76,7 \% aller Fehler) aus, bei den professionellen Übersetzern sind es durchschnittlich mit 9 Fehlern ebenfalls 72,6 \% der Gesamtfehlerzahl. Für die Didaktik kann hieraus gefolgert werden, dass der Verbesserung des semantischen Differenzierungsvermögens mehr Beachtung geschenkt werden muss.

Wendet man die in Abschnitt 3 spezifizierten Indikatoren für Problemstellen an, so haben Studierende mit durchschnittlich 36,3 Problemen fast dreimal so viele wie professionelle Übersetzer mit durchschnittlich nur 13,6. Diese Differenz muss aber vor dem Hintergrund der Datenerhebungsmethoden gesehen werden: Mit zunehmender translatorischer Kompetenz laufen mehr und mehr Prozesse automatisiert ab und stehen damit nach dem Informationsverarbeitungsmodell von Ericsson/Simon (1993) nicht zur Verbalisierung zur Verfügung. Folglich hinterlassen automatisierte Problemlösungsprozesse auch keine Spuren in den Übersetzungsprotokollen und scheinen in den Statistiken nicht auf. Die Entlastung, die die Automatisierung mit sich bringt, kann jedoch bei den professionellen Übersetzern durchaus Gedächtniskapazität für die Lösung komplexerer Probleme freisetzen. So stellten z. B. bereits Jääskeläinen/Tirkkonen-Condit (1991) fest, dass sich die Probleme, die Gegenstand bewusster Entscheidungsprozesse werden, mit zunehmender translatorischer Kompetenz verändern, also nicht unbedingt abnehmen. Dies steht in Einklang mit Ergebnissen weiterer Translationswissenschaftler, wie z. B. Gerloff (1988: 54 ff.), aber auch mit Befunden aus der kognitionswissenschaftlichen Expertiseforschung (z. B. Dreyfus/Dreyfus 1986; Ericsson/Smith 1991: 25 f.; Anderson 2007), aus der bekannt ist, dass mit zunehmender Expertise mehr Faktoren in Problemlösungsprozesse einbezogen werden. Dieser Einbezug zusätzlicher Faktoren wie beispielsweise des größeren Kontexts führt zu einer stärkeren Auslastung des Arbeitsgedächtnisses, was wiederum zur Folge haben kann, dass für das laute Denken keine kognitive Kapazität mehr zur Verfügung steht und so komplexe Probleme zwar gelöst werden, in den Übersetzungsprotokollen aber ebenfalls keine Spuren hinterlassen. Berücksichtigt man dies, so muss man davon ausgehen, dass die Diskrepanz zwischen der Anzahl der Problemstellen, auf die die Studierenden stießen, und derjenigen der professionellen Übersetzer geringer ausfällt, als dies aus den Übersetzungsprotokollen hervorgeht, aber auch, dass sie anderer Art sind, was in Abschnitt 5.2 näher untersucht wird.

Wenn professionelle Übersetzer mehr Faktoren bei der Suche nach Übersetzungslösungen gleichzeitig berücksichtigen können (und damit stärker ko- und kontextorientiert vorgehen), erklärt dies auch, dass die professionellen Übersetzer im Vergleich zu den Novizen durchschnittlich kürzere Revisionsphasen (post-phases) aufwiesen (durchschnittlich 8,5 Minuten oder 18,7 \% der Gesamtübersetzungsdauer bei den professionellen Übersetzern vs. 19,2 Minuten oder 26,6 \% der Gesamtübersetzungsdauer bei den Novizen), dafür aber etwas mehr Zeit in die Hauptphasen investierten (durchschnittlich 52,3 Minuten oder 72,3 \% der Gesamtübersetzungsdauer bei den Novizen vs. 35,3 Minuten oder 77,8 \% der Gesamtübersetzungsdauer bei den professionellen Übersetzern). Es liegt hier die Vermutung nahe, dass die Studierenden, um der Komplexität des Übersetzungsvorgangs Herr zu werden, in der Hauptphase bestimmte Aspekte ausblenden oder verschieben, um eine Komplexitätsreduktion zu erreichen, die dann aber dazu führt, dass viele Probleme später in der Revisionsphase gelöst werden müssen. Diese Vermutung steht im Einklang mit der Beobachtung, dass Novizen die Lösung von Problemstellen viel häufiger verschleppen als professionelle Übersetzer (siehe unten). Sie deckt sich aber nicht mit den Beobachtungen von Jakobsen (2002), der feststellte, dass professionelle Übersetzer mehr Zeit auf die Orientierungsphase (pre-phase) und die Revisionsphase verwendeten als seine semi-professionellen Übersetzer (vgl. hierzu referierend auch Göpferich 2008: 176). Wie die Revisionsphase fiel auch die Orientierungsphase bei den Studierenden mit durchschnittlich 4 Minuten oder 5,5 \% der Ge- 
samtübersetzungsdauer länger aus als bei den professionellen Übersetzern mit durchschnittlich nur 1,6 Minuten oder 3,5 \% der Gesamtübersetzungsdauer.

Eine Korrelation zwischen der Übersetzungsdauer und der Produktqualität kann innerhalb der Gruppen nicht eindeutig festgestellt werden. Zwar weisen die beiden besten studentischen Vpn (BKR und SFR) mit jeweils 127 bzw. 87 Minuten die längste Versuchsdauer und zugleich mit 10 bzw. 14,5 Fehlern die niedrigste Fehlerzahl im ZT auf und die schlechteste studentische Vpn mit 30,5 Fehler auch die kürzeste Versuchsdauer (32 Minuten). Bei den professionellen Vpn hingegen wurde die niedrigste Fehlerzahl (9,5 bzw. 11 Fehler) von den beiden Vpn AEF und AIR erzielt, von denen AIR mit der Übersetzung am schnellsten fertig war (in 35 Minuten), AEF aber die zweitlängste Dauer (57 Minuten) aufweist.

Betrachtet man die in den Übersetzungsprotokollen nachweisbaren Probleme näher, so fällt auf, dass die professionellen Übersetzer Probleme fast immer in einem Arbeitsgang lösten. Von den bei allen Professionellen zusammen insgesamt 68 aufgetretenen Problemen wurden nur 5 verschleppt, d. h. zunächst ungelöst gelassen und später wieder aufgenommen, und dies auch nur von zwei Vpn (s. die Rubrik „Problem incidents and types“ in den Tabellen in Göpferich 2009b). Diese verschleppten von den 5 Wiederaufnahmen dann 1 nochmals. Bei den Studierenden hingegen werden von den 218 insgesamt bei ihnen aufgetretenen Problemen 52 verschleppt, von diesen 52 dann nochmals 13, von diesen 13 dann in einem dritten Durchgang nochmals 6 und von diesen 6 nochmals 1. Solche Verschleppungen treten bei allen sechs studentischen Vpn auf; bei zwei von ihnen werden Probleme zweifach verschleppt, bei zwei dreifach und bei zwei sogar vier- bzw. fünffach. Dieses Verschleppen könnte ein Anzeichen dafür sein, dass Probleme nicht zielgerichtet gelöst werden und an die Stelle von Strategien andere ineffiziente Lösungsansätze treten, die auch reines Raten oder ,Sich-auf-sein-Gefühl-Verlassen’ beinhalten, was die Detailanalysen zu Problemlösungswegen in Abschnitt 5.2 bestätigen. Vor diesem Hintergrund muss die Tatsache, dass die Studierenden mit 67,5 \% ihrer Probleme fast eine genauso hohe Problemlösungsquote haben wie die professionellen Vpn mit 69,1 \% wohl eher auf glückliches Raten als auf strategische Problemlösungskompetenz zurückgeführt werden und kann damit nicht als Indikator für Kompetenz betrachtet werden. ${ }^{3}$

Die Anzahl der Wiedergabeprobleme und kombinierten Verstehens- und Wiedergabeprobleme ist bei den Studierenden mit durchschnittlich $17(11,8+5,2)$ bzw. 15,3 $(8,8+6,5)$ mehr als doppelt bzw. dreimal so hoch wie bei den professionellen Übersetzern mit durchschnittlich nur 8,2 $(6,4+1,8)$ bzw. 4,4 $(2,2+2,2)$. Die Anzahl der Verstehensprobleme ist bei den Studierenden mit durchschnittlich $4(3,8+0,2)$ sogar fünfmal so hoch wie bei den professionellen Übersetzern mit durchschnittlich nur 0,8 (0,6 + 0,2). Dass die professionellen Übersetzer deutlich weniger Verstehensprobleme haben als die Novizen, dürfte noch primär mit der fremdsprachlichen Kompetenz in Zusammenhang stehen und muss kein Indikator für eine schwächere translatorische Kompetenz sein.

Der Schwierigkeitsgrad des Textes wurde von den Studierenden auf einer fünfstufigen Skala (1= niedrig bis $5=$ sehr hoch) mit durchschnittlich 3,8 deutlich höher bewertet als von den professionellen Übersetzern mit durchschnittlich nur 2,3 (s. hierzu die Rubrik „Estimated text difficulty“ in den Tabellen in Göpferich 2009b).

3 Bei der nahezu gleich hohen Problemlösungsquote von Novizen und professionellen Übersetzern ist ferner zu beachten, dass diese Quote nur den Problemlösungserfolg an denjenigen Stellen berücksichtigt, an denen die Vpn den verwendeten Problemindikatoren zufolge ein Problem hatten (s. Abschnitt 3). Nicht berücksichtigt sind hierin die Stellen, an denen die Vpn Fehler machten, ohne sich auch nur bewusst gewesen zu sein, dass die betreffende Stelle problematisch sein könnte. Darüber hinaus muss auch beachtet werden, dass die Fehler aus funktionaler Perspektive unterschiedlich gravierend ausgefallen sein können, beispielsweise gravierender bei den Novizen als bei den professionellen Übersetzern. Beide Erklärungsansätze können im Rahmen dieses Artikels jedoch nicht weiter verfolgt werden. 


\subsection{Zielgerichtetheit des Problemlöseverhaltens}

Im Folgenden soll der Problemlösungsweg für drei ausgewählte Probleme aufgezeigt werden. Sie wurden ausgewählt, weil sie erstens den meisten Vpn besondere Schwierigkeiten bereitet haben und zweitens ganz unterschiedlicher Natur sind. Beim ersten Problem (control switch) handelt es sich um ein terminologisches Problem, beim zweiten (You can stand the appliance on its base while you are using it.) um einen Defekt oder zumindest eine missverständliche Stelle im AT und beim dritten (Angabe der Einsatzmöglichkeiten für die verschiedenen Geschwindigkeitsstufen) um ein Problem im Bereich der Kulturspezifik.

\subsection{1. control switch}

Vier der fünf Übersetzungsprotokolle der professionellen Übersetzer (AEF, KEG, LEB, RAN) und fünf der sechs Übersetzungsprotokolle der Novizen (BKR, KNI, SFR, HHE, KBE) enthalten die Problemstelle control switch. Die professionellen Übersetzerinnen AEF und LEB und die Novizin SFR nennen control switch auch im versuchsbegleitenden Fragebogen als Problemstelle. Lediglich das Übersetzungsprotokoll und der versuchsbegleitende Fragebogen des Profis AIR und der Novizin TDI weisen keine Anzeichen dafür auf, dass sie ein Problem bei der Übersetzung von control switch empfanden; AIR hatte objektiv jedoch dennoch ein Problem, denn die Übersetzung Ein-Aus-Schalter, für die er sich entscheidet, ist, wie ich noch aufzeigen werde, falsch.

Die Tatsache, dass die Übertragung von control switch also allen fünf professionellen Übersetzern und fünf der sechs Novizen Schwierigkeiten bereitet hat, ist der Grund dafür, dass die Ansätze der Vpn, dieses Problem zu lösen, hier näher betrachtet werden sollen. Ein weiterer Grund ist, dass nur drei der fünf professionellen Vpn eine korrekte Lösung gewählt haben; bei den Studierenden waren es immerhin vier von sechs.

\section{Professionelle Vpn:}

Tab. 1 informiert über die von den professionellen Vpn gewählten deutschen Entsprechungen von control switch, die von ihnen erwogenen Alternativen (sofern vorhanden) und ihre Kommentare zu diesem Problem im versuchsbegleitenden Fragebogen sowie den von ihnen eingeschlagenen Lösungsweg. Tab. 2 unten liefert die entsprechenden Informationen für die studentischen Vpn. Der Problemlösungsweg der Vpn in der letzten Spalte der Tabellen lässt erkennen oder zumindest erahnen, inwiefern die Vpn zielgerichtet und kriteriengeleitet, also strategisch, vorgegangen sind und inwiefern nicht. Hieraus lässt sich wiederum bis zu einem gewissen Grad schließen, inwiefern eine korrekte Lösung nur als Zufallstreffer betrachtet werden kann und damit kein Zeichen translatorischer Kompetenz ist oder aber als Lösung, die strategisch erarbeitet wurde, d. h. notwendige Kriterien erfüllt, deren Erfüllung von der Vpn bewusst angestrebt wurde. 


\begin{tabular}{|c|c|c|c|}
\hline Vpn & " Version im ZT & $\begin{array}{l}\text { in Erwägung gezogene } \\
\text { Alternativen (auch in } \\
\text { Wörterbüchern gefundene } \\
\text { Äquivalente) } \\
\text { Kommentare im } \\
\text { versuchsbegleitenden } \\
\text { Fragebogen }\end{array}$ & Lösungsansatz \\
\hline AEF & $\begin{array}{l}\text { Bedienungsschalter D } \\
\text { (akzeptabel) }\end{array}$ & $\begin{array}{l}\text { Betriebsschalter } \\
\text { Regelschalter } \\
\text { „Man verwendet zwar einen } \\
\text { Mixer und andere Geräte } \\
\text { sehr oft, kann aber die } \\
\text { Bestandteile oft nicht } \\
\text { benennen.“ } \\
\text { Vpn ist mit ihrer Lösung } \\
\text { „eher zufrieden“. }\end{array}$ & $\begin{array}{l}\mathrm{P}^{4} \\
\text { assoziiert Bedienungsschalter und Betriebsschalter }(+)^{5} \\
\text { sucht „,ontrol-switch deutsch“ erfolglos in Google (-) } \\
\text { schlägt control switch im zweisprachigen Technik- } \\
\text { Fachwörterbuch Ernst nach (-) } \\
\text { assoziiert Regelschalter (+) } \\
\text { sucht „Regelschalter Mixer“ und „Bedienungsschalter } \\
\text { Mixer“ in Google, um festzustellen, ob diese } \\
\text { Bezeichnungen im Zusammenhang mit Mixern } \\
\text { verwendet werden (0) } \\
\text { entscheidet sich ohne Angabe von Gründen für } \\
\text { Bedienungsschalter (0) }\end{array}$ \\
\hline AIR & $\begin{array}{l}\text { Ein-Aus-Schalter D } \\
\text { (nicht akzeptabel) }\end{array}$ & $\begin{array}{l}\text { keine Erwähnung im } \\
\text { versuchsbegleitenden } \\
\text { Fragebogen }\end{array}$ & $\begin{array}{l}\text { keine hinreichenden Problemstellenindikatoren } \\
\text { konsultiert Abbildungen in der Anleitung, um Aussehen } \\
\text { des Schalters herauszufinden }(+) \\
\text { folgert, dass es der Ein-Aus-Schalter sein muss, weil das } \\
\text { Gerät sonst keinen entsprechenden Schalter hat }(0)\end{array}$ \\
\hline KEG & $\begin{array}{l}\text { Schalter D } \\
\text { (akzeptabel) }\end{array}$ & $\begin{array}{l}\text { Hauptschalter } \\
\text { Sicherheitsschalter } \\
\text { keine Erwähnung im } \\
\text { versuchsbegleitenden } \\
\text { Fragebogen }\end{array}$ & $\begin{array}{l}\text { P1, 1.2, 1.3 } \\
\text { äußert Ziel der Benennung nach der Funktion (+) } \\
\text { um diese herauszufinden, hätte er gerne das Gerät zur } \\
\text { Verfügung }(+) \\
\text { schließt aus Kotext (vermutlich „With the control switch } \\
\text { (D) on 0, the attachments can be removed ...“), dass es } \\
\text { sich um einen Hauptschalter handeln muss, womit die } \\
\text { Funktion geklärt und das Rezeptionsproblem gelöst ist (+) } \\
\text { - Das Wiedergabeproblem wird aber noch aufgeschoben. } \\
\text { entdeckt in dem nicht zu übersetzenden Kotext, der } \\
\text { mitgeliefert wurde, die Information „Speeds selected with } \\
\text { control switch“ und schließt hieraus korrekt, dass es nur } \\
\text { einen Schalter gibt, den er daher auch nur Schalter nennt } \\
\text { und damit auch das Wiedergabeproblem löst (+) }\end{array}$ \\
\hline LEB & $\begin{array}{l}\text { Kontrollschalter D } \\
\text { (nicht akzeptabel) }\end{array}$ & $\begin{array}{l}\text { keine } \\
\text { „Definitionsproblem [sic]: } \\
\text { Wie sagen } \\
\text { Mixerhersteller“? } \\
\text { Vpn ist mit ihrer Lösung } \\
\text { „eher unzufrieden“. }\end{array}$ & $\begin{array}{l}\text { P2 } \\
\text { fragt sich, um was für einen Schalter es sich handelt, und } \\
\text { konsultiert zur Beantwortung die Aufgabenstellung mit } \\
\text { den Abbildungen (+) } \\
\text { konsultiert im Internet Texte von Elektrogeräteherstellern, } \\
\text { um an die einschlägige Terminologie zu gelangen, die sie } \\
\text { als die Hauptschwierigkeit des Textes betrachtet (-) } \\
\text { wählt aber eine an die Form des AS-Ausdrucks angelehnte } \\
\text { Benennung, die eindeutig falsch ist, da der Schalter nichts } \\
\text { kontrolliert (-) }\end{array}$ \\
\hline RAN & $\begin{array}{l}\text { Regler D } \\
\text { (akzeptabel) }\end{array}$ & $\begin{array}{l}\text { Steuerregler } \\
\text { Schieber } \\
\text { Knopf } \\
\text { keine Erwähnung im } \\
\text { versuchsbegleitenden } \\
\text { Fragebogen }\end{array}$ & $\begin{array}{l}\text { P3 } \\
\text { assoziiert sofort Steuerregler, fragt sich aber, wie die ent- } \\
\text { sprechende Bedieneinheit üblicherweise genannt wird (+) } \\
\text { stellt keine gezielten Problemlösungsversuche an (-) } \\
\text { entscheidet sich für Regler: „schreib ma einfach nur } \\
\text { Regler“ (Risikovermeidungsstrategie?) (0) }\end{array}$ \\
\hline
\end{tabular}

Tab. 1. Übersetzungsproblem „control switch“ (professionelle Übersetzer)

4 Diese Angabe bezieht sich auf die Nummerierung der Problemstellen in den Übersetzungsprotokollen. Folgt der ersten Ziffer nach dem P (für Problemstelle) nach einem Punkt noch eine zweite, so gibt diese die Wiederaufnahme, also das Verschleppen des Problems, an (.2 = erste Wiederaufnahme, . 3 = zweite Wiederaufnahme etc.).

5 In den Tab. 1 bis 6 kennzeichnen +-Zeichen sinnvolle und zielführende Maßnahmen und Entscheidungen, --Zeichen nicht sinnvolle und nicht zielgerichtete Maßnahmen sowie falsche Entscheidungen und 0-Zeichen Entscheidungen, die zwar durchdacht sein mögen, aber dennoch zu kurz greifen, oder aber, bei denen nicht entschieden werden kann, ob sie vom Zufall geleitet waren und dann eher negativ zu werten wären oder aber strategisch erfolgten und dann positiv verbucht werden müssten. 
Betrachten wir zuerst den Lösungsweg derjenigen professionellen Übersetzer, in deren ZT eine korrekte Lösung erscheint.

AEF hat zwei primäre Äquivalentassoziationen (Bedienungsschalter und Betriebsschalter), hinsichtlich deren Angemessenheit sie sich aber unsicher ist. Um sich zu vergewissern, ob es die von ihr assoziierten deutschen Termini gibt, schlägt sie im zweisprachigen Technik-Fachwörterbuch Ernst nach, ein unsinniges Unterfangen, da der Schalter nach seiner Form oder Funktion bei dem konkreten Mixer benannt werden muss und ein Wörterbuch hier keine Hilfe bieten kann. Auch die Suche der Kombinationen „Regelschalter Mixer“ und „Bedienungsschalter Mixer“ in Google kann nicht als sinnvolle Maßnahme betrachtet werden. Sie mag zwar Auskunft darüber geben, ob diese Bezeichnungen im Zusammenhang mit Mixern gebraucht werden, wobei von einer positiven Antwort hierauf aber nicht darauf geschlossen werden kann, dass der entsprechende Regler auch bei dem Mixer, dessen Anleitung übersetzt werden soll, so benannt werden kann, denn er kann ja durchaus auch Zusatzfunktionen haben, die zu berücksichtigen wären, und muss ggf. im Licht anderer Bedienungselemente gesehen werden, die der Mixer auch noch aufweist und von denen der zu benennende Regler auch durch seine Bezeichnung klar unterschieden werden muss. Die Entscheidung, die AEF am Ende fällt, erfolgt also nicht anhand klarer Kriterien; zumindest sind solche in den Verbalisierungen nicht zu erkennen. Dennoch gibt AEF im versuchsbegleitenden Fragebogen an, dass sie mit ihrer Lösung „eher zufrieden“ ist, was sie letztendlich auch sein kann. AEF errät die Lösung nicht einfach, sondern stellt, wie oben aufgezeigt, durchaus sinnvolle Überlegungen an, die jedoch am Ende zu kurz greifen, so dass der Lösungsweg nicht als effizient und optimal begründet betrachtet werden kann. Anders ist dies bei KEG.

Wie aus seinem Lösungsweg hervorgeht, verfolgt KEG eine klare Strategie im in Abschnitt 4.2 definierten Sinne. Er strebt eine Benennung nach der Funktion des Schalters an und möchte diese herausfinden, wozu er gerne das Gerät zur Verfügung hätte und mangels Verfügbarkeit auf den Kotext mit Abbildungen zurückgreift. Auf diese Weise findet er die Funktion des Schalters heraus (Hauptschalter), entscheidet sich aber für das in seinem Kotext hinreichend spezifizierte Hyperonym Schalter, da es der einzige Schalter des Gerätes ist und daher in Abgrenzung von den anderen Bedienungselementen, bei denen es sich um Tasten ${ }^{6}$ handelt, nicht weiter spezifiziert werden muss. KEGs Lösungsweg enthält keine überflüssigen Schritte und ausschließlich begründete Entscheidungen. Er geht optimal effizient vor.

RAN liefert zwar auch eine korrekte Lösung, bei ihr bleibt jedoch unklar, ob es sich hierbei um Zufall handelt oder ob sie z. B. unsicher ist und eine Risikovermeidungsstrategie verfolgt, was immerhin auch ein strategisches Vorgehen wäre. Bei ihrer primären Äquivalentassoziation Steuerregler ist sie unsicher, ob das die übliche Benennung für den entsprechenden Schalter eines Mixers ist; sie stellt jedoch keine gezielten Problemlösungsversuche an. Ich vermute hier, dass sie glaubt, man müsse das entsprechende Wissen einfach haben, was bei ihr nicht der Fall ist, und sie deshalb nicht motiviert ist, Strategien zu entwickeln, mit denen sich eine sichere Lösung erarbeiten ließe, was als Defizit in ihrer Problemlösungskompetenz zu betrachten ist.

Betrachten wir nun die Lösungswege der nicht erfolgreichen professionellen Übersetzer:

AIR verfehlt zwar das Ziel einer korrekten Lösung im ZT; die Überlegungen, die er anstellt, sind jedoch strategisch ausgerichtet, und er verfehlt eine Lösung nur knapp, da lediglich seine letzte Überlegung zu kurz greift: Dass es einen Ein-Aus-Schalter geben muss, ist völlig richtig. Dieser kann aber außer dem Ein-Aus-Schalten noch weitere Funktionen haben, was bei dem Mixer auch der Fall ist. AIR scheitert also nur deshalb, weil er diese letzte Eigenschaft des Schalters nicht bedenkt. Insofern lässt sein Vorgehen auf mehr translatorische Kompetenz schließen als

6 Für den Fachmann kann auch eine Taste (ein Taster) ein Schalter sein, wenn man mit ihr (ihm) durch Niederdrücken etwas ein- und ausschalten kann. Für technische Laien (und die zu übersetzende Anleitung richtet sich an solche) ist ein prototypischer Schalter jedoch ein Wippschalter oder ein Stufenschalter wie bei dem Mixer, um den es in der Anleitung geht. 
beispielsweise das der professionellen Übersetzerin RAN und das der Novizin TDI, die zwar eine korrekte Lösung vorzuweisen haben, bei denen es aber keine Indikatoren dafür gibt, dass sie diese wirklich auf strategischem Wege gefunden haben.

LEB ergreift zwar zunächst eine zielführende Maßnahme: Sie fragt sich, um was für eine Art von Schalter es sich handelt, und sucht die Lösung im Kotext mit den Abbildungen. Dass sie dann aber im Internet Texte von Elektrogeräteherstellern konsultiert, um an die einschlägige Terminologie zu gelangen, greift wiederum zu kurz, denn so mag sie zwar übliche Bezeichnungen für Bedienungselemente von Elektrogeräten herausfinden, ob eine davon und welche dann zu dem zu dokumentierenden Mixer passt, darauf gibt diese Recherche mit Sicherheit keine Antwort. Dass sie schließlich die an die Form des englischen Ausdrucks angelehnte deutsche Bezeichnung Kontrollschalter wählt, die völlig unsinnig ist, weil der Schalter nichts ,kontrolliert', legt die Vermutung nahe, dass die Vpn sich zu wenig selbst zutraut, eine sinnvolle Benennung auf der Basis dessen, was sie im Text über den Schalter erfährt, zu kreieren. Sie wählt schließlich eine eng an die englische Bezeichnung angelehnte deutsche Benennung, mit der sie laut Angaben im versuchsbegleitenden Fragebogen „eher unzufrieden“ ist. Hier liegt die Vermutung nahe, dass sie nicht nach Kriterien sucht, die die Lösung erfüllen muss, sondern eine vermeintliche Absicherungsstrategie ergreift, die darin besteht, dass sie nah am AT bleibt, was eine typische Vorgehensweise von Novizen ist, einer professionellen Übersetzerin völlig unwürdig und bei ihr auf eine relativ geringe translatorische Kompetenz schließen lässt.

Alles in allem liefern von den fünf professionellen Übersetzern zwar nur drei eine korrekte Lösung, doch immerhin lassen auch drei - wenn auch nicht dieselben - eine strategische Vorgehensweise erkennen.

\section{Novizen:}

\begin{tabular}{|c|c|c|c|}
\hline Vpn & Version im ZT & $\begin{array}{l}\text { in Erwägung } \\
\text { gezogene Alternativen } \\
\text { (auch in } \\
\text { Wörterbüchern } \\
\text { gefundene } \\
\text { Äquivalente) } \\
\text { Kommentare im } \\
\text { versuchsbegleitenden } \\
\text { Fragebogen }\end{array}$ & Lösungsansatz \\
\hline BKR & $\begin{array}{l}\text { Geschwindigkeits- } \\
\text { regler D } \\
\text { (akzeptabel) }\end{array}$ & $\begin{array}{l}\text { Regulierungsschalter } \\
\text { Bedienknopf } \\
\text { Bedienungselement } \\
\text { Bedienelement } \\
\text { Bedienschalter/-knopf } \\
\text { Bedienungsschalter } \\
\text { Steuerungsschalter } \\
\text { Steuerschalter } \\
\text { Taster } \\
\text { Knopf } \\
\text { Einschaltknopf } \\
\text { für sich selbst: „der } \\
\text { Stöpsel da“ } \\
\text { keine Erwähnung im } \\
\text { versuchsbegleitenden } \\
\text { Fragebogen }\end{array}$ & $\begin{array}{l}\text { P2, 2.2, 2.3, } 2.4 \\
\text { informiert sich über das Aussehen in den } \\
\text { Abbildungen der Anleitung (+) } \\
\text { sucht nach üblichen deutschen Bezeichnungen und } \\
\text { überlegt, in welchen externen Ressourcen sie am } \\
\text { ehesten fündig werden könnte (+) } \\
\text { wählt vorläufig bis zur Revisionsphase Schalter D als } \\
\text { Übersetzung mit der Begründung, dass man über das } \\
D \text { schon herausfinden kann, welches Bedienungs- } \\
\text { element gemeint ist (+) } \\
\text { konsultiert einsprachige englische Wörterbücher und } \\
\text { ein Synonymwörterbuch für das Deutsche (-) } \\
\text { wünscht sich eine Terminologiedatenbank (+) } \\
\text { Vpn fällt, evtl. inspiriert durch in Nachschlagewerken } \\
\text { gefundene Benennungen, Geschwindigkeitsregler } \\
\text { ein, was sie extrem positiv bewertet, ohne Gründe } \\
\text { dafür anzugeben (+) }\end{array}$ \\
\hline
\end{tabular}




\begin{tabular}{|c|c|c|c|}
\hline KNI & $\begin{array}{l}\text { Hauptschalter D } \\
\text { (akzeptabel) }\end{array}$ & $\begin{array}{l}\text { Kontrollknopf } \\
\text { Hauptschalter } \\
\text { Bedienungsschalter } \\
\text { Steuerschalter } \\
\text { keine Erwähnung im } \\
\text { versuchsbegleitenden } \\
\text { Fragebogen }\end{array}$ & $\begin{array}{l}\text { P4, } 4.2 \\
\text { sucht nach üblicher deutscher Benennung für control } \\
\text { switch und assoziiert spontan Kontrollknopf und } \\
\text { Hauptschalter, von denen sie eher Hauptschalter in } \\
\text { Erwägung zieht (0) } \\
\text { schlägt control switch im zweisprachigen Online- } \\
\text { Wörterbuch Leo nach, wo sie verschiedene } \\
\text { Äquivalente liest, u. a. Hauptschalter, wodurch sie } \\
\text { sich in ihrer Präferenz bestätigt zu fühlen scheint, } \\
\text { obwohl diese Benennung in ihren Ohren nicht gut } \\
\text { klingt (-) } \\
\text { Vpn setzt sich über dieses Geschmacksurteil hinweg } \\
\text { mit der Begründung, dass Wohlklang keine } \\
\text { Anforderung an das gesuchte ZS-Äquivalent ist (+) }\end{array}$ \\
\hline SFR & $\begin{array}{l}\text { Bedienungs- } \\
\text { schalter D } \\
\text { (akzeptabel) }\end{array}$ & $\begin{array}{l}\text { Bedienschalter } \\
\text { Steuerschalter } \\
\text { Handknopf } \\
\text { Automatikschalter } \\
\text { Hauptschalter } \\
\text { Zeitschalter } \\
\text { Ein-Aus-Schalter } \\
\text { indirekte Erwähnung im } \\
\text { versuchsbegleitenden } \\
\text { Fragebogen: „Technische } \\
\text { Begriffe (z. B. button, } \\
\text { switch ....“/Schwierig- } \\
\text { keit: „Begriffe für die } \\
\text { technischen Dinge wie } \\
\text { z. B. button zu finden, } \\
\text { die so im Deutschen } \\
\text { existieren und die ein } \\
\text { deutschsprachiger Leser } \\
\text { auch verstehen würde.“ }\end{array}$ & $\begin{array}{l}\text { P2 } \\
\text { stellt fest, dass mit control switch ein bestimmter } \\
\text { Schalter gemeint ist, ist sich aber bei dessen } \\
\text { Spezifizierung (control) unsicher (+) } \\
\text { schlägt im zweisprachigen Online-Wörterbuch Leo } \\
\text { control switch nach und liest verschiedene } \\
\text { angebotene Äquivalente (-) } \\
\text { aus diesen greift sie ohne Angabe von Gründen } \\
\text { Bedienungsschalter heraus (0) } \\
\text { sie befindet die Benennung Bedienungsschalter als } \\
\text { „komisch“ und fragt sich, wie man den Schalter beim } \\
\text { Mixer nennt (+) } \\
\text { Vpn erwägt verschiedene Äquivalentassoziationen, } \\
\text { die sie hat, sowie Übersetzungsäquivalente, die sie in } \\
\text { Leo vorfindet (+) } \\
\text { Vpn entscheidet sich ohne Angabe von Gründen für } \\
\text { Bedienungschalter, was sie wie folgt vor sich selbst } \\
\text { legitimiert: „wenn man das vorher definiert, dass } \\
\text { Bedienungsschalter dieses D ist, dann heißt das halt } \\
\text { so“ (+) }\end{array}$ \\
\hline HHE & $\begin{array}{l}\text { Kontrollschalter } \\
\text { D } \\
\text { (nicht akzeptabel) }\end{array}$ & $\begin{array}{l}\text { Bedienschalter } \\
\text { Kontrollschalter } \\
\text { Steuerschalter } \\
\text { Bedienungsschalter } \\
\text { keine Erwähnung im } \\
\text { versuchsbegleitenden } \\
\text { Fragebogen }\end{array}$ & $\begin{array}{l}\text { P1 } \\
\text { schlägt control switch im zweisprachigen Online- } \\
\text { Wörterbuch Leo nach und liest verschiedene dort } \\
\text { angeboten Äquivalente, u.a. Steuerschalter und } \\
\text { Kontrollschalter (+) } \\
\text { lehnt Steuerschalter ab mit der wenig fundierten } \\
\text { Begründung, es klinge nicht gut (,Na, das hurcht si } \\
\text { Topfen an“) (-) } \\
\text { verwendet ohne Angabe von Gründen und Kriterien } \\
\text { Kontrollschalter (-) }\end{array}$ \\
\hline TDI & $\begin{array}{l}\text { Schalter D } \\
\text { (akzeptabel) }\end{array}$ & $\begin{array}{l}\text { Kontrollschalter } \\
\text { Schalter } \\
\text { keine Erwähnung im } \\
\text { versuchsbegleitenden } \\
\text { Fragebogen }\end{array}$ & $\begin{array}{l}\text { keine hinreichenden Problemindikatoren } \\
\text { assoziiert spontan als Übersetzung für control switch } \\
\text { Kontrollschalter und beginnt auch, sich dies zu } \\
\text { diktieren, schreibt dann aber nur Schalter, ohne dies } \\
\text { zu begründen (Risikovermeidungsstrategie?) (0) }\end{array}$ \\
\hline KBE & $\begin{array}{l}\text { Kontrollschalter } \\
\text { D } \\
\text { (nicht akzeptabel) }\end{array}$ & $\begin{array}{l}\text { keine } \\
\text { keine Erwähnung im } \\
\text { versuchsbegleitenden } \\
\text { Fragebogen }\end{array}$ & $\begin{array}{l}\text { P2 } \\
\text { schlägt switch im zweisprachigen Online-Wörterbuch } \\
\text { dict.cc nach und entscheidet sich für Schalter }(+) \\
\text { verwendet dann ohne weitere Recherche Kontroll- } \\
\text { schalter, ohne zu hinterfragen, ob diese Benennung } \\
\text { semantisch in ihrem Kontext sinnvoll sein kann }(-)\end{array}$ \\
\hline
\end{tabular}

Tab. 2. Übersetzungsproblem „,control switch“ (Novizen) 
Betrachten wir auch hier wieder zunächst die Lösungswege der Vpn mit korrekten deutschen Entsprechungen für control switch. Hierbei handelt es sich um BKR, KNI, SFR und TDI. Bei BKR, KNI und SFR stellen die korrekten Lösungen mit hoher Wahrscheinlichkeit keine Zufallstreffer dar, im Gegensatz zur korrekten Lösung von TDI, deren Übersetzungsprotokoll keine Indikatoren dafür enthält, dass sie sich der Kriterien bewusst gewesen wäre, die das deutsche Äquivalent von control switch erfüllen muss. Ihre einzige Entscheidung, die strategisch durchdacht sein könnte, ist anstelle ihrer primären Äquivalentassoziation Kontrollschalter nur Schalter zu verwenden. Der Grund hierfür mag gewesen sein, dass sie sich hinsichtlich des Attributs Kontroll- unsicher war, sie aber mit Sicherheit sagen konnte, dass es sich bei dem zu benennenden Bedienungselement um einen Schalter handelt. Dass sie schließlich nur Schalter verwendet, könnte eine Risikovermeidungsstrategie sein. Ob TDI den Ausdruck Kontrollschalter letztlich bewusst und kriteriengeleitet verwirft, ist auf der Grundlage seines Übersetzungsprotokolls nicht zu beurteilen.

BKR geht sehr zielgerichtet vor (s. Tab. 2). Nur einer ihrer Schritte, die Suche in einsprachigen englischen Wörterbüchern und einem deutschen Synonymwörterbuch, ist nicht zielführend und kann als Zeitvergeudung betrachtet werden. Ihre Kriterien sind die Benennung des Schalters nach dem Aussehen, die eindeutige Identifizierbarkeit für den Benutzer und die Konventionalität der Bezeichnung. Dass sie sich für Geschwindigkeitsregler entscheidet, könnte ein Zeichen dafür sein, dass sie den Schalter auch nach der Funktion benennen will, was sich aber aus ihren Aussagen nicht mit Bestimmtheit schließen lässt. Obwohl es sich bei BKR um eine Übersetzungsnovizin im ersten Semester handelt, kann ihre Vorgehensweise durchaus als kompetent bezeichnet werden.

Aus den Aussagen von KNI lässt sich trotz ihrer korrekten Lösung nicht klar schließen, ob sie sich wirklich der Kriterien bewusst ist, die die gesuchte Übersetzung für control switch erfüllen muss. Das einzige Kriterium, das sie explizit verbalisiert, ist, dass Wohlklang nicht zu den Anforderungen an die gesuchte Lösung gehört.

Wie bei KNI weiß man auch bei SFR nicht, ob sie sich der Anforderungen, die die Lösung erfüllen muss, bewusst ist. Bewusst ist sie sich jedoch der Tatsache, dass die von ihr gewählte Lösung akzeptabel ist, weil durch den Bezugsbuchstaben $D$ für den Leser die Identifikation des Schalters eindeutig möglich ist.

Während sich TDI noch der Tatsache bewusst gewesen sein könnte, dass der fragliche Schalter nichts kontrolliert, ist dieses Bewusstsein bei KBE nicht vorhanden. Sie verwendet völlig unreflektiert Kontrollschalter, was auf eine Interferenz control - Kontrolle zurückzuführen sein könnte.

Wie KBE verwendet auch HHE Kontrollschalter, ohne sich der Unsinnigkeit der Benennung bewusst zu sein. Der einzige aus dem Übersetzungsprotokoll zu erschließende Grund für die Wahl dieser Benennung ist, dass ihr diese Bezeichnung besser zu gefallen scheint als der ebenfalls in Erwägung gezogene Ausdruck Steuerschalter.

Bei zwei der sechs studentischen Vpn (KBE und HHE) ist bei der Übersetzung von control switch also keinerlei strategisches Vorgehen und damit auch keine Problemlösungskompetenz zu erkennen. Zwei Novizen (BKR und SFR) zeigen ein hohes Maß an Problemlösungskompetenz und zwei weitere (KNI und TDI) lassen Ansätze strategischen Vorgehens erkennen.

Insgesamt erstaunt, dass die Novizen häufiger korrekte Lösungen hervorgebracht haben als die professionellen Übersetzer. Dennoch sind die Lösungswege der professionellen Übersetzer strategischer ausgerichtet. Immerhin gibt es unter den professionellen Übersetzern einen (KEG) mit einem optimalen Lösungsweg. Dies ist zwar auch bei einer Novizin (BKR) der Fall, deren Lösungsweg büßt aber durch nicht zielführende Schritte an Effizienz ein. Ein völlig strategieloses Vorgehen ist bei keiner der professionellen Vpn zu beobachten, unter den Novizen aber bei immerhin zweien (HHE und KBE).

Betrachten wir nun, wie man idealerweise zu einer akzeptablen Übersetzungslösung kommt: Ein Fachübersetzer, der auf Technik spezialisiert ist, assoziiert control nicht mit Kontrolle wie 
z. B. LEB, HHE, TDI und KBE, sondern weiß, dass to control in technischen Kontexten steuern (ohne Rückkoppelung) oder regeln (mit Rückkoppelung) oder allgemeiner bedienen bedeutet, während das deutsche kontrollieren im Englischen in der Regel mit to monitor zum Ausdruck gebracht wird. Aus Verständlichkeitsgründen empfiehlt sich eine Benennung des Schalters nach der Form und/oder der Funktion. Um sich über diese zu informieren, empfiehlt es sich, die mitgelieferten Abbildungen und, wenn diese allein nicht ausreichen, auch den Kotext zu konsultieren. Aus den Abbildungen geht eindeutig hervor, wie der Schalter aussieht; durch Verwendung des Referenzbuchstabens kann für den Leser ein eindeutiger Bezug zu dem Schalter hergestellt werden, was alle Vpn machen. Aus dem Kotext geht auch hervor, dass derselbe Schalter nicht nur zum Ein- und Ausschalten, sondern auch zur Wahl der drei Geschwindigkeitsstufen benutzt wird. Alle anderen Bedienungselemente sind, wie aus den Abbildungen und dem Kotext ebenfalls hervorgeht, Tasten. Als Benennungen nach der Form ergeben sich somit als mögliche deutsche Äquivalente Schalter und Regler. Diese können nach der Funktion spezifiziert werden, müssen dies aber nicht, z. B. als Geschwindigkeitsregler, Hauptschalter oder Bedienungsschalter, wobei letzteres zwar akzeptabel, aber redundant ist, denn mit jedem Schalter wird etwas bedient; ein Schalter ist ein Bedienungselement.

\subsubsection{You can stand the appliance on its base while you are using it}

Diese Textstelle bereitete den Vpn aus unterschiedlichen Gründen Schwierigkeiten. Diese waren erstens terminologischer Art (base, appliance) und wurden zweitens verursacht durch die Unlogik oder zumindest Missverständlichkeit der AT-Textstelle: Man kann den Mixer nicht auf seine Standfläche stellen, während man ihn benutzt (,while you are using it“), sondern allenfalls während man ihn zwar im Einsatz (d. h. aus dem Schrank geholt) hat, aber gerade nicht damit mixt. Nur das letztere Problem soll hier näher behandelt werden, wobei aber auch die terminologischen Probleme nicht völlig unberücksichtigt bleiben können, weil sie zu einer Überlagerung von Problemen haben führen können, die die Aufmerksamkeit der Vpn von dem uns hier interessierenden Problem evtl. abgezogen haben könnten in dem Sinne, dass die Vpn das Problem der Unlogik vielleicht hätten lösen können, wenn sie an der betreffenden Stelle nicht durch andere Probleme abgelenkt worden wären.

Für die in der Überschrift genannte Textstelle liefern nur zwei der fünf professionellen Übersetzer (LEB und RAN) und nur eine einzige Studierende (BKR) eine akzeptable Lösung. Allerdings erkennen zwei weitere professionelle Übersetzer (AIR und KEG) die Unlogik des Ausgangstextes und beschließen, den Hersteller hierzu zu befragen, was ebenfalls als strategisch sinnvolles Vorgehen betrachtet werden muss. Von den Studierenden hingegen erkennt nur eine (BKR) die Unlogik und versucht sie aufzulösen; bei allen anderen Studierenden finden sich keine Hinweise in den Daten, dass sie die Unlogik der Textstelle erkannt hätten.

Es kann als Zeichen translatorischer Kompetenz betrachtet werden, dass der Übersetzer nicht einfach wiedergibt, was im AT steht, sondern diesen auch unter dem Gesichtspunkt der Logik und Korrektheit hinterfragt. Erfreulicherweise ist dies bei vier der fünf professionellen Übersetzer der Fall. Sehr positiv zu bewerten ist, dass auch eine Novizin (BKR) bereits entsprechende Überlegungen anstellt und dabei zu einer Lösung kommt, die auf jeden Fall richtig sein muss, ohne dass sie sie mit dem Hersteller besprechen müsste. Der professionelle Übersetzer KEG und die Novizin BKR nehmen darüber hinaus auch noch eine Textoptimierung vor, indem sie den Bezugsbuchstaben $G$ aus der Abbildung, der im AT nicht vorhanden ist, einfügen. Beide verfolgen einen fast optimalen strategischen Problemlösungsweg ohne nicht (potenziell) zielführende Maßnahmen, fast deshalb, weil BKRs Problemlösungsweg einen nicht zielführenden und daher überflüssigen Schritt enthält (s. Tab. 4).

In den versuchsbegleitenden Fragebögen thematisieren die professionellen Übersetzer nur die Übersetzung von base als Problem. Dies ist auch bei einer Studentin (TDI) der Fall, zwei Studentinnen (BKR, HHE) nennen while you are using it ebenfalls als Problem. Lediglich die professionelle Übersetzerin LEB thematisiert die Textstelle weder im versuchsbegleitenden Fragebogen, 
noch enthält ihr Übersetzungsprotokoll entsprechende Problemindikatoren. Sie liefert aber eine korrekte Lösung.

\begin{tabular}{|c|c|c|c|}
\hline Vpn & Version im ZT & $\begin{array}{l}\text { in Erwägung gezogene } \\
\text { Alternativen (auch in } \\
\text { Wörterbüchern } \\
\text { gefundene Äquivalente) } \\
\text { Kommentare im } \\
\text { versuchsbegleitenden } \\
\text { Fragebogen }\end{array}$ & Lösungsansatz \\
\hline $\mathrm{AEF}$ & $\begin{array}{l}\text { Das Gerät kann } \\
\text { während des } \\
\text { Betriebs aufgestellt } \\
\text { sein. } \\
\text { (nicht akzeptabel) }\end{array}$ & $\begin{array}{l}\text { kann während des } \\
\text { Betriebs aufgestellt } \\
\text { werden } \\
\text { kann während des } \\
\text { Betriebs auf seiner Basis } \\
\text { stehen } \\
\text { stand on its base - „Die } \\
\text {,base’ ist im Text nicht } \\
\text { definiert [sic: sie ist aber } \\
\text { in der Abb. zu sehen], da } \\
\text { es aber nur eine mögliche } \\
\text { Stellung gibt, habe ich } \\
\text { einfach ,aufstellen’ } \\
\text { verwendet.” } \\
\text { Vpn ist mit ihrer Lösung } \\
\text { „eher zufrieden“, was sie } \\
\text { in Bezug auf die Über- } \\
\text { setzung von base auch } \\
\text { sein kann, aber nicht in } \\
\text { Bezug auf die Aussage } \\
\text { des Gesamtsatzes. }\end{array}$ & $\begin{array}{l}\text { P3; P3.2, P18 } \\
\text { Vpn ist nicht klar, was mit base gemeint ist (+) } \\
\text { erschließt aus ihrem Weltwissen korrekt, was gemeint sein } \\
\text { muss, was sie anhand der Abb. hätte verifizieren können, } \\
\text { aber nicht tut (+) } \\
\text { Die Daten lassen keinen Rückschluss darauf zu, dass die Vpn } \\
\text { die Unlogik der Textstelle reflektiert. (-) }\end{array}$ \\
\hline AIR & $\begin{array}{l}\text { Während Sie das } \\
\text { Gerät benutzen, } \\
\text { können Sie es auf } \\
\text { seine Unterseite } \\
\text { stellen. } \\
\text { (nicht akzeptabel) }\end{array}$ & $\begin{array}{l}\text { zu sich selbst: Unterteil } \\
\text { Grundfläche } \\
\text { Unterseite } \\
\text { Sie können das Gerät } \\
\text { während der Benutzung } \\
\text { auf seine Unterseite } \\
\text { stellen. } \\
\text { base - „dachte zuerst an } \\
\text {,Basis-Station’. War aber } \\
\text { natürlich völlig falsch. } \\
\text { Vpn ist mit ihrer Lösung } \\
\text { „sehr zufrieden“, weil der } \\
\text { „Begriff technisch } \\
\text { korrekt“ sei. In Bezug auf } \\
\text { die Übersetzung von base } \\
\text { kann sie das auch sein, } \\
\text { nicht aber in Bezug auf } \\
\text { die Aussage des } \\
\text { Gesamtsatzes. }\end{array}$ & $\begin{array}{l}\text { P3 } \\
\text { Vpn fragt sich, was mit base gemeint ist (+) } \\
\text { konsultiert die Abb., in der die base nicht explizit bezeichnet } \\
\text { ist (aber indirekt über die non-slip rubber feet), und vermutet } \\
\text { richtig, es handelt sich dabei um den „Unterteil“ (+) } \\
\text { Vpn versucht, sich den Sachverhalt vorzustellen, und meldet } \\
\text { Zweifel an der Logik an: ,aber er wird ja wohl net den Mixer } \\
\text { so halten, und dann müsst er das ja in der Luft halten“ (+) } \\
\text { diese Unlogik veranlasst die Vpn, erneut darüber } \\
\text { nachzudenken, ob sie base richtig verstanden hat (+) } \\
\text { Vpn assoziiert Ladebasisstation „,wie bei einem Handy“, was } \\
\text { sie aber gleich wieder als „Blödsinn“ verwirft (+) } \\
\text { Vpn konsultiert erneut Abb. (+) } \\
\text { Vpn äußert die Absicht, den Hersteller zu befragen (+) } \\
\text { die im ZT erscheinende Version gefällt der Vpn } \\
\text { berechtigterweise nicht (+) } \\
\text { Vpn nominalisiert den Temporalsatz (,während der } \\
\text { Benutzung“), befindet das Resultat aber nicht als besser (,,is } \\
\text { ja wurscht“) (+) } \\
\text { Vpn bewertet den Teil „das Gerät auf seine Unterseite“ } \\
\text { positiv (+) }\end{array}$ \\
\hline KEG & $\begin{array}{l}\text { Wenn sie [sic] das } \\
\text { Gerät benutzen, } \\
\text { können Sie es auf } \\
\text { seine Grundplatte } \\
\text { (G) stellen. ??? } \\
\text { (nicht akzeptabel) }\end{array}$ & $\begin{array}{l}\text { keine } \\
\text { keine Erwähnung im } \\
\text { versuchsbegleitenden } \\
\text { Fragebogen }\end{array}$ & $\begin{array}{l}\text { P3, P3.2 } \\
\text { Vpn fragt sich, was mit base gemeint ist (die Seite, auf der } \\
\text { die Knethaken sind, oder die gegenüber liegende) }(+) \\
\text { beantwortet die Frage anhand der Abb., in der mit } G \text { die } \\
\text { „non-slip rubber feet (on base)“ beschriftet sind: Grundplatte } \\
\text { (+) } \\
\text { Vpn optimiert Text durch Einfügen des Bezugsbuchstabens } \\
\text { G, der im AT nicht erscheint (+) } \\
\text { Vpn erkennt, dass der AT keinen Sinn ergibt: „Des is aber } \\
\text { irgendwie witzig, weil ja dann der Teig aus ... der Schüssel } \\
\text { herausfallt, aber so steht’s da!“ (+) }\end{array}$ \\
\hline
\end{tabular}

7 Für die Bewertung als „akzeptabel“ bzw. „nicht akzeptabel“ war hier nur das Kriterium ausschlaggebend, ob die Unlogik/Missverständlichkeit der Textstelle behoben wurde. Fehler anderer Art blieben unberücksichtigt. 


\begin{tabular}{|c|c|c|c|}
\hline & & & $\begin{array}{l}\text { Vpn übersetzt dennoch wörtlich, versieht den ZT aber mit } \\
\text { drei Fragezeichen, mit denen sie kennzeichnet, dass hier eine } \\
\text { Rückfrage an den Auftraggeber erforderlich ist. (+) } \\
\text { Vpn kommentiert die Unlogik des AT nochmals in der } \\
\text { Revisionsphase, ohne Änderungen vorzunehmen: „Das } \\
\text { könnat ma im Weltraum probieren, wo der Teig nicht unter } \\
\text { der Schwerkraft leidet.“ (+) } \\
\text { Vpn verweist nochmals auf die Notwendigkeit der } \\
\text { Rücksprache mit dem Auftraggeber (+) }\end{array}$ \\
\hline LEB & $\begin{array}{l}\text { Sie können das } \\
\text { Gerät beim } \\
\text { Arbeiten auf die } \\
\text { Standfläche } \\
\text { aufstellen [sic]. } \\
\text { (akzeptabel) }\end{array}$ & $\begin{array}{l}\text { Sie können das Gerät auf } \\
\text { die Standfläche aufstellen, } \\
\text { während sie es benutzen. } \\
\text { (Zwischenlösung in } \\
\text { Translog) } \\
\text { keine Erwähnung im } \\
\text { versuchsbegleitenden } \\
\text { Fragebogen }\end{array}$ & $\begin{array}{l}\text { keine hinreichenden Problemindikatoren } \\
\text { keine verbalisierten Reflexionen zur fraglichen Textstelle } \\
\text { nicht beurteilbar, ob strategisch (Änderung in die Endversion } \\
\text { erst in der Revisionsphase) }\end{array}$ \\
\hline RAN & $\begin{array}{l}\text { Der Mixer kann } \\
\text { zwischendurch } \\
\text { senkrecht } \\
\text { aufgestellt } \\
\text { werden. } \\
\text { (akzeptabel; sehr } \\
\text { gute Lösung) }\end{array}$ & $\begin{array}{l}\text { Der Mixer kann aufgestellt } \\
\text { werden. } \\
\text { Der Mixer kann } \\
\text { zwischendurch aufgestellt } \\
\text { werden. (beides Zwischen- } \\
\text { lösungen in Translog) } \\
\text { stand the appliance on its } \\
\text { base while you are using it } \\
\text { - Schwierigkeit bestand in: } \\
\text {,ein Wort oder eine } \\
\text { Formulierung zu finden, die } \\
\text { genau das präzise } \\
\text { ausdrückt, was ich } \\
\text { ausdrücken wollte (Bild war } \\
\text { klar!)” }\end{array}$ & $\begin{array}{l}\text { keine hinreichenden Problemindikatoren } \\
\text { keine verbalisierten Reflexionen zur fraglichen Textstelle } \\
\text { nicht eindeutig beurteilbar, ob strategisch, aber sehr } \\
\text { wahrscheinlich, da notwendige Veränderung gegenüber AT }\end{array}$ \\
\hline
\end{tabular}

Tab. 3. Übersetzungsproblem „stand ... on its base while you are using it” (professionelle Übersetzer)

\begin{tabular}{|c|c|c|c|}
\hline Vpn & Version im ZT & $\begin{array}{l}\text { in Erwägung gezogene } \\
\text { Alternativen (auch in } \\
\text { Wörterbüchern gefundene } \\
\text { Äquivalente) } \\
\text { Kommentare im } \\
\text { versuchsbegleitenden } \\
\text { Fragebogen }\end{array}$ & Lösungsansatz \\
\hline BKR & $\begin{array}{l}\text { Sie können das } \\
\text { Gerät auf seine } \\
\text { Standfläche G } \\
\text { stellen, wenn Sie } \\
\text { es gerade nicht } \\
\text { verwenden. } \\
\text { (akzeptabel; sehr } \\
\text { gute Lösung) }\end{array}$ & $\begin{array}{l}\text { Sie können das Gerät auf } \\
\text { seine Standfläche G stellen, } \\
\text { während Sie es nicht } \\
\text { verwenden. } \\
\text { while you are using it - Vpn } \\
\text { ist mit dem Ergebnis „,eher } \\
\text { zufrieden“. }\end{array}$ & $\begin{array}{l}\text { P13, P13.2. P14, P14.2, P14.3 } \\
\text { Vpn stockt schon in der Orientierungsphase beim Lesen der } \\
\text { betreffenden Textstelle und versucht sich den Sachverhalt } \\
\text { vorzustellen (+) } \\
\text { ist unsicher, ob es sich um einen Handmixer oder eine } \\
\text { Küchenmaschine handelt; in diesem Zusammenhang kommt } \\
\text { sie auf den fraglichen Satz zurück (+) } \\
\text { schlägt base im zweisprachigen Online-Wörterbuch Leo } \\
\text { nach, dessen Lösungsangebote sie verwirft (+) } \\
\text { konsultiert stattdessen die Abb. und klärt damit das } \\
\text { Verstehensproblem (+) } \\
\text { wundert sich, dass man das Gerät hinstellen können soll, } \\
\text { während man es verwendet (+) } \\
\text { geht davon aus, dass man das Gerät eher hinstellen kann, } \\
\text { wenn man es gerade nicht braucht (+) } \\
\text { denkt über passende deutsche Entsprechung für base nach } \\
\text { (sie weiß genau, was gemeint ist) und konsultiert Leo erneut } \\
(-) \\
\text { entscheidet sich für Standfläche und fügt zur Herstellung } \\
\text { eines eindeutigen Bezugs den Bezugsbuchstaben G aus der } \\
\text { Abb. in den ZT ein (+) } \\
\text { wundert sich, dass genau das Gegenteil von dem gemeint sein } \\
\text { muss, was im AT steht, erwägt aber auch, dass sie den Text } \\
\text { falsch verstanden haben könnte (,Na, weil wenn i’s während } \\
\text { verwend, dann kann i’s net hinstellen, weil dann quirln die }\end{array}$ \\
\hline
\end{tabular}




\begin{tabular}{|c|c|c|c|}
\hline & & & $\begin{array}{l}\text { Mixerquirl in der Luft herum. Während Sie es NICHT verwenden } \\
\text { müssat ma sagn. Aber das is dann nit mehr das Gleiche. While } \\
\text { you are using it - während Sie es nicht verwenden - des is (da } \\
\text { jetz) das komplette Gegenteil, aber es is vül sinnvoller, i kann, } \\
\text { ach vielleicht hab i’s falsch verstanden. Schau ma nachher nach.“ } \\
(+) \\
\text { kommt in Revisionsphase auf das Problem zurück und versucht } \\
\text { ihr Verständnis mit der Semantik des AT in Einklang zu bringen } \\
\text { (während man das Gerät aus dem Schrank geholt, aber gerade } \\
\text { nicht im Einsatz hat), was mit hoher Wahrscheinlichkeit im AT } \\
\text { gemeint ist }(+)\end{array}$ \\
\hline KNI & $\begin{array}{l}\text { Sie können das } \\
\text { Gerät während des } \\
\text { Gebrauchs auf die } \\
\text { Halterung [sic] } \\
\text { stellen. } \\
\text { (nicht akzeptabel) }\end{array}$ & $\begin{array}{l}\text { Sie können das Gerät auf } \\
\text { seine Halterung stellen, } \\
\text { während Sie es verwenden. } \\
\text { Sie können das Gerät } \\
\text { während des Gebrauchs auf } \\
\text { der Halterung abstellen. } \\
\text { keine Erwähnung im } \\
\text { versuchsbegleitenden } \\
\text { Fragebogen }\end{array}$ & $\begin{array}{l}\text { P9, P9.2, P10 } \\
\text { assoziiert: „Sie können das Gerät auf seine Halterung stellen, } \\
\text { während Sie es verwenden“, ist aber unsicher, ob sie base richtig } \\
\text { verstanden hat (+) } \\
\text { sucht base in der Abb., wird aber nicht fündig (+), obwohl man } \\
\text { sie indirekt über die non-slip rubber feet identifizieren kann } \\
\text { schlägt base im zweisprachigen Online-Wörterbuch Leo nach (-) } \\
\text { vermutet nach wie vor, dass es um eine Halterung geht (-) } \\
\text { reflektiert die grammatische Einbettung von Halterung (auf die } \\
\text { oder auf der Halterung abstellen) (+) } \\
\text { Die Daten lassen keinen Rückschluss darauf zu, dass die Vpn die } \\
\text { Unlogik der Textstelle reflektiert. (-) }\end{array}$ \\
\hline SFR & $\begin{array}{l}\text { Sie können das } \\
\text { Gerät auf seine } \\
\text { Standfläche } \\
\text { stellen, während } \\
\text { Sie es benutzen. } \\
\text { (nicht akzeptabel) }\end{array}$ & $\begin{array}{l}\text { Sie können das Gerät } \\
\text { hinstellen, während Sie es } \\
\text { benützen. } \\
\text { keine Erwähnung im } \\
\text { versuchsbegleitenden } \\
\text { Fragebogen }\end{array}$ & $\begin{array}{l}\text { P8 } \\
\text { assoziiert zunächst die in Erwägung gezogene Alternative (+) } \\
\text { ist unsicher, was mit base gemeint ist und konsultiert das } \\
\text { zweisprachige Online-Wörterbuch Leo, mit dessen } \\
\text { Lösungsvorschlägen sie nicht zufrieden ist }(+) \\
\text { konsultiert Kotext mit Abb., womit sie das Verstehensproblem } \\
\text { löst (+) } \\
\text { Die Daten lassen keinen Rückschluss darauf zu, dass die Vpn die } \\
\text { Unlogik der Textstelle reflektiert. (-) }\end{array}$ \\
\hline HHE & $\begin{array}{l}\text { Sie können das } \\
\text { Gerät während des } \\
\text { Gebrauchs auf die } \\
\text { Seitenfläche [sic] } \\
\text { stellen. } \\
\text { (nicht akzeptabel) }\end{array}$ & $\begin{array}{l}\text { Standfläche } \\
\text { Arbeitsgrundlage } \\
\text { Boden } \\
\text { Bodenplatte } \\
\text { Grundfläche } \\
\text { auf den Kopf stellen } \\
\text { You can stand the appliance } \\
\text { on its base while you are } \\
\text { using it. - Vpn ist mit dem } \\
\text { Ergebnis ,eher } \\
\text { unzufrieden“. }\end{array}$ & $\begin{array}{l}\text { P10, P11; P11.2 } \\
\text { schlägt base in zweisprachigen Online-Wörterbuch Leo nach (+) } \\
\text { fragt sich, was der ganze Satz bedeuten soll (+) } \\
\text { sucht in Google Seiten zu Mixern (+) } \\
\text { schreibt „Sie können das Gerät während des Gebrauchs“ und } \\
\text { lässt den Rest offen bis in die Revisionsphase (0) } \\
\text { kommentiert die Version im ZT, die die Vpn erst in der } \\
\text { Revisionsphase niederschreibt, mit „naja, lass ma’s so“ (-) } \\
\text { Die Daten lassen keinen eindeutigen Rückschluss darauf zu, dass } \\
\text { die Vpn die Unlogik der Textstelle reflektiert. (-) }\end{array}$ \\
\hline TDI & $\begin{array}{l}\text { Sie können die } \\
\text { Gerätschaft [sic] } \\
\text { während dem } \\
\text { Gebrauch [sic] auf } \\
\text { die Standfläche } \\
\text { stellen. } \\
\text { (nicht akzeptabel) }\end{array}$ & $\begin{array}{l}\text { Sie können die Gerätschaft } \\
\text { während dem Gebrauch auf } \\
\text { ihre Basis stellen. } \\
\text { auf den Fuß stellen } \\
\text { hinstellen } \\
\text { Boden } \\
\text { Fundament } \\
\text { Grundplatte } \\
\text { Fußgestell } \\
\text { base - Vpn ist mit Ergebnis } \\
\text { „eher zufrieden“. }\end{array}$ & $\begin{array}{l}\text { P6, P7; P7.2 } \\
\text { assoziiert zunächst die in Erwägung gezogene Alternative (+) } \\
\text { schlägt base in zweisprachigen Online-Wörterbuch Leo nach (+) } \\
\text { formuliert Alternativen für stand on its base }(+) \\
\text { denkt über den Kasus nach, der nach während folgen muss, ohne } \\
\text { zu korrektem Ergebnis zu kommen (+) } \\
\text { schlägt base erneut in zweisprachigen Online-Wörterbuch Leo } \\
\text { nach (-) } \\
\text { erwägt andere Lösungen für base, die sich für sie alle nicht so gut } \\
\text { anhören (+) } \\
\text { informiert sich über das Aussehen der base in den Abb. }(+) \\
\text { bewertet die Alternative auf die Standfläche als passend (+) } \\
\text { Die Daten lassen keinen Rückschluss darauf zu, dass die Vpn die } \\
\text { Unlogik der Textstelle reflektiert. }(-)\end{array}$ \\
\hline $\mathrm{KBE}$ & $\begin{array}{l}\text { Sie können das } \\
\text { Gerät stehen } \\
\text { lassen [sic], } \\
\text { während Sie es } \\
\text { benützen. } \\
\text { (nicht akzeptabel) }\end{array}$ & $\begin{array}{l}\text { Sie können das Gerät auf } \\
\text { seinem Grund/Boden stehen } \\
\text { lassen, während Sie es } \\
\text { benützen. } \\
\text { keine Erwähnung im } \\
\text { versuchsbegleitenden } \\
\text { Fragebogen }\end{array}$ & $\begin{array}{l}\text { P8 } \\
\text { schlägt base in zweisprachigen Online-Wörterbuch dict.cc nach } \\
\text { und findet u.a. Grund (+) } \\
\text { schreibt die in Erwägung gezogene Alternative nieder (-) } \\
\text { kommentiert Boden in der Revisionsphase als „Bledsinn“ und } \\
\text { entfernt es schließlich (-) } \\
\text { Die Daten lassen keinen Rückschluss darauf zu, dass die Vpn die } \\
\text { Unlogik der Textstelle reflektiert. (-) }\end{array}$ \\
\hline
\end{tabular}

Tab. 4. Übersetzungsproblem „stand ... on its base while you are using it” (Novizen) 
Auf die Vorstellung eines idealen Problemlösungsweges kann bei diesem Beispiel verzichtet werden, da der Profi KEG und die Novizin BKR (BKR mit Ausnahme eines Schrittes) einen solchen einschlagen.

Im Zusammenhang mit diesem Übersetzungsbeispiel, das eine Optimierung des Inhalts des Textes erforderte, erscheint es interessant, auch einen Blick zu werfen auf die übrigen in Abschnitt 4.2 vorgestellten Überlegungen, die ein idealer Übersetzer anstellen sollte, um zu einer unter funktionalen Gesichtspunkten adäquaten Übersetzungslösung zu gelangen. Von den sechs notwendigen Überlegungen wurden von den professionellen Übersetzern nachweisbar im Schnitt 2,4 angestellt, von den Novizen jedoch nur 1,2; von den wünschenswerten waren es bei den professionellen Übersetzern im Schnitt 0,4 von 3, bei den Novizen nur 0,2 von 3 (s. hierzu im Detail die Rubrik „Reflections“ in den Tab. 1 und 2 in Göpferich 2009b). Diese Befunde deuten darauf hin, dass die professionellen Übersetzer eine kritischere Distanz zum AT einnehmen und globalere Strategien verfolgen als die Novizen, eine Beobachtung, denen in zukünftigen Detailanalysen weiter nachgegangen werden soll.

\subsubsection{Beispiele für Einsatzmöglichkeiten der verschiedenen Geschwindigkeitsstufen}

Für die Geschwindigkeitsstufen 2 und 3 des Mixers werden jeweils vier Beispiele für Mixgut genannt, für das die jeweilige Geschwindigkeitsstufe verwendet werden kann. Die Übersetzung einiger dieser Beispiele ist insofern schwierig, als es um kulturspezifische Gerichte geht (pancake) oder aber Entsprechungen im Deutschen gefunden werden müssen, die in allen deutschsprachigen Ländern verstanden werden, wobei es gerade bei Speisen kulturelle Unterschiede in den Benennungen gibt (Schlagsahne und Omelett in Deutschland vs. Schlagobers und Palatschinke in Österreich). Der ideale Übersetzer erkennt, dass es bei der Übersetzung der Beispiele nicht auf Denotatsgleichheit ankommt, sondern lediglich darauf, für beide Geschwindigkeitsstufen typische Beispiele für den deutschsprachigen Raum zu finden, wobei aus rhetorischen Gründen sogar jeweils drei Beispiele ausreichen, obwohl es im AT jeweils vier sind. Entsprechungen für die Stufe 2 müssen außerdem das Kriterium der relativen Dünnflüssigkeit erfüllen, Entsprechungen für die Stufe 3 das Kriterium der relativen Zähflüssigkeit und der Notwendigkeit einer sehr feinen Vermischung der Bestandteile bzw. eines intensiven Verwirbelns. Außerdem müssen die aufgezählten Mixgüter denselben Abstraktionsgrad haben; es können als Beispiele also nicht Kuchenteig und Rührteig nebeneinander genannt werden, da auch Kuchenteig in der Regel ein Rührteig ist. ${ }^{8}$

Betrachten wir nun, inwiefern die Vpn diese Kriterien in die Findung deutscher Entsprechungen einbezogen haben.

Keine der Vpn, auch keine der professionellen, reduziert die Beispiele auf jeweils drei oder lässt in ihrem Verhalten oder ihren Äußerungen darauf schließen, dass ihr das Kriterium der nicht notwendigen Denotatsgleichheit bewusst gewesen wäre. Dies ist insofern bedauerlich, als die Berücksichtigung dieser Kriterien den Vpn viel Zeit beim Nachschlagen erspart und somit zu einem effizienteren Übersetzungsprozess geführt hätte, mit dem sich zudem noch viele Fehler hätten vermeiden lassen.

Der Kulturspezifik von engl. pancake sind sich immerhin eine professionelle Übersetzerin (AEF) und eine Novizin (BKR) bewusst, wobei weitere Vpn diese Kulturspezifik erkannt haben mögen, ihre Daten liefern jedoch keine Hinweise darauf.

Hinsichtlich der Problematik der bundesdeutschen oder österreichischen Variante liefern alle Vpn mit Ausnahme des professionellen Übersetzers AIR insofern korrekte Lösungen, als sie sich für eine der beiden Varianten, meist die bundesdeutsche, entscheiden. Lediglich AIR liefert be-

8 Es kann hier zur Diskussion gestellt werden, ob Kuchenteig und Hefeteig in einer Auszählung erscheinen können, da Hefeteig auch als Kuchenteig verwendet werden kann. Die drei Begutachter der ZT haben diese Kombination aber nicht als Fehler gewertet, weil man mit Kuchenteig prototypischerweise einen Rührteig assoziiert. 
wusst einen Mix (Pfannkuchenteig und Schlagobers), wobei seine Äußerungen auf einen gewissen Nationalstolz schließen lassen. Explizit reflektiert wird diese Problematik von vier der fünf professionellen Übersetzer (alle außer LEB), aber nur zwei der studentischen (BKR und KNI). Die Novizin SFR ist die einzige deutsche Studentin, die vermutlich deshalb nicht reflektiert, weil die Verwendung der bundesdeutschen Variante für sie im Gegensatz zu den Österreichern der ,Normalfall’ ist.

Das Kriterium, dass die Elemente der Aufzählungen auf derselben Abstraktionsstufe stehen müssen, wird von den ZT aller professionellen Übersetzer mit Ausnahme desjenigen von AEF erfüllt. Sie stellt Kuchenteig, Rührteig und Knetteig in eine Aufzählung, wobei Knetteig in Opposition zu Rührteig eine gute Lösung darstellt, Kuchenteig jedoch insofern disparat ist, als ein Kuchenteig sowohl ein Rühr- als auch ein Knetteig sein kann. Auch die ZT von fünf der sechs Novizen erfüllen die Anforderung, HHE stellt jedoch Kuchenteig, Teig und schweren Teig in eine Aufzählung, wobei Teig ein Hyperonym ist und streng genommen auch ein Kuchenteig ein schwerer Teig sein kann.

Insgesamt liefern, wenn man nur die o. g. genannten Kriterien (s. auch Fußnote 9) zur Beurteilung heranzieht, zwei von fünf professionellen Übersetzern und vier von sechs Novizen eine akzeptable Lösung, wobei die professionellen Übersetzer jedoch effizienter zum Ziel kommen. Ihre Effizienz zeigt sich vor allen in der deutlich geringeren Anzahl Nachschlagevorgänge, die in den Tab. 5 und 6 jeweils in der dritten Spalte mit angegeben sind. Im Schnitt beträgt die Anzahl der Nachschlagevorgänge für die Übersetzung der Beispiele bei den Professionellen 2,4 pro Vpn, wobei keine Wiederaufnahmen desselben Problems vorkommen, bei den Studierenden hingegen 6 pro Vpn, wobei die Anzahl der Wiederaufnahmen bis zu 3 (bei BKR und KNI) beträgt. Hierbei ist zwar wiederum zu beachten, dass die Studierenden allein schon aufgrund ihrer geringeren fremdsprachlichen Kompetenz häufiger nachschlagen müssen, um Verstehensprobleme zu lösen, dennoch zeigt ein Vergleich der Lösungsschritte in den Tab. 5 und 6, dass die Studierenden auch an vielen Stellen nicht zielführend nachschlagen oder aber, um sich abzusichern, ohne dass dies erforderlich gewesen wäre.

\begin{tabular}{|c|c|c|c|}
\hline Vpn & Version im ZT & $\begin{array}{l}\text { in Erwägung gezogene } \\
\text { Alternativen (auch in } \\
\text { Wörterbüchern gefundene } \\
\text { Äquivalente) } \\
\text { Kommentare im } \\
\text { versuchsbegleitenden } \\
\text { Fragebogen }\end{array}$ & Lösungsansatz \\
\hline $\mathrm{AEF}$ & $\begin{array}{l}\text { 2: Omelettenteig } \\
\text { [sic], Schlagrahm, } \\
\text { Mayonnaise, } \\
\text { Soßen } \\
\text { 3: Eiklar, } \\
\text { Kuchenteig, } \\
\text { Rührteig [sic], } \\
\text { Knetteig }\end{array}$ & $\begin{array}{l}\text { schwerer Teig } \\
\text { Brotteig } \\
\text { Eiklar } \\
\text { Rührkuchenteig } \\
\text { pancake - Vpn ist mit ihrer } \\
\text { Lösung „eher zufrieden“. }\end{array}$ & $\begin{array}{l}\text { P9 (pancake), P10 (batter), P11 (pastry), P12 (heavy dough) - } \\
4 \text { Nachschlagevorgänge } \\
\text { Vpn fragt sich, was das europäische Pendant von pancake ist, } \\
\text { ist sich also der Kulturspezifik bewusst }(+)^{10} \\
\text { entscheidet sich ohne Nachschlagen für Omelettenteig [sic], } \\
\text { weil diese Benennung ,am unverfänglichsten“ ist, womit sie } \\
\text { sich wahrscheinlich auf die überregionale Verständlichkeit } \\
\text { bezieht (+) }\end{array}$ \\
\hline
\end{tabular}

9 Für die Bewertung als „,akzeptabel“ bzw. „nicht akzeptabel“ werden hier nur die Kriterien Verständlichkeit im deutschsprachigen Raum, Dünn- bzw. Dickflüssigkeit und Gleichrangigkeit der aufgezählten Satzglieder herangezogen. Rechtschreib-, Numerus- und Kollokationsfehler werden nicht betrachtet. Obwohl an Stellen, an denen es keine im gesamten deutschsprachigen Raum verwendeten Bezeichnungen gibt, die bundesdeutschen vorzuziehen wären, weil die Bundesdeutschen die größte Sprechergruppe ausmachen, wird auch akzeptiert, wenn sich eine Vpn aus Nationalstolz bewusst (auch) für eine österreichische Benennung entscheidet, zumal die entsprechenden Benennungen auch in Deutschland verstanden werden.

10 Gezählt werden in den Tab. 5 und 6 nur diejenigen Nachschlagevorgänge, die sich auf die zu übersetzenden Wörter beziehen, wobei dasselbe Item nur einmal gezählt wird, auch wenn es mehrfach nachgeschlagen wurde. Nicht einbezogen werden z. B. Recherchen zur Orthographie und zu weiteren Wörtern, die evtl. in Explikationen angetroffen wurden. 


\begin{tabular}{|c|c|c|c|}
\hline & $\begin{array}{l}\text { (nicht akzeptabel, } \\
\text { weil Kuchenteig in } \\
\text { der Regel ein } \\
\text { Rührteig ist) }^{11}\end{array}$ & & $\begin{array}{l}\text { Vpn ist sich der Bedeutung von batter nicht sicher und } \\
\text { schlägt „,batter deutsch“ in Google nach, stellt dann aber fest, } \\
\text { dass Omelettenteig [sic] ohnehin ausreicht (+) } \\
\text { entscheidet sich für die bundesdeutschen Ausdrücke } \\
\text { (Schlagrahm statt Schlagobers) (+) } \\
\text { Vpn ist sich der Bedeutung von pastry unsicher und schlägt } \\
\text { u.a. im zweisprachigen Wörterbuch dict.cc nach, obwohl sie } \\
\text { weiß, dass es auch um einen Teig gehen muss (-) } \\
\text { Vpn ist sich der Bedeutung von heavy dough unsicher und } \\
\text { schlägt dough u.a. im zweisprachigen Wörterbuch dict.cc } \\
\text { nach (-), verlässt sich bei ihrer Entscheidung aber auf ihre } \\
\text { eigenen Assoziationen (+) } \\
\text { ändert in der Revisionsphase Rührkuchenteig in Kuchenteig } \\
\text { ab und ergänzt Rührteig „zum Unterschied von dem andern“, } \\
\text { ohne sich bewusst zu sein, dass Kuchenteige meist Rührteige } \\
\text { sind und so eine disparate Aufzählung entsteht (-) }\end{array}$ \\
\hline AIR & $\begin{array}{l}\text { 2: Pfannkuchenteig, } \\
\text { Schlagobers, } \\
\text { Mayonnaise, } \\
\text { Saucen } \\
\text { 3: Eiklar, } \\
\text { Kuchenback- } \\
\text { mischung [sic], } \\
\text { Gebäck }^{12} \text {, schwerer } \\
\text { Teig } \\
\text { (akzeptabel) }\end{array}$ & $\begin{array}{l}\text { Kuchenmischung } \\
\text { Kuchenteig } \\
\text { keine Erwähnung im } \\
\text { versuchsbegleitenden } \\
\text { Fragebogen }\end{array}$ & $\begin{array}{l}\text { P7 (pancake batter), P8 (cake mix), P9 (pastry), P10 (heavy } \\
\text { dough) - } 4 \text { Nachschlagevorgänge } \\
\text { erkennt das Problem der unterschiedlichen Benennungen in } \\
\text { den deutschsprachigen Ländern und entscheidet sich zunächst } \\
\text { für die bundesdeutschen Varianten (+), ist darin aber nicht } \\
\text { konsequent und verwendet - sozusagen zum Ausgleich - } \\
\text { auch den österreichischen Ausdruck Schlagobers (,Ja, ich } \\
\text { müsst den Kunden fragen, weil die Deutschen verstehen } \\
\text { wahrscheinlich den Schlagobers net. Aber wenn i schon } \\
\text { Pfannkuchen schreiben muss, dann schreib i wenigstens } \\
\text { Schlagobers.“) } \\
\text { ist sich der Bedeutung von cake mix nicht sicher und schlägt } \\
\text { in Google nach, wo er Backmischung findet, was er als } \\
\text { passend bewertet (+) } \\
\text { schlägt pastry im zweisprachigen Langenscheidt-Wörterbuch } \\
\text { nach, obwohl er in etwa weiß, was es bedeuten muss (-) } \\
\text { assoziiert mit heavy dough schwerer Teig (+) } \\
\text { schlägt heavy dough in Google.at nach (-) } \\
\text { schlägt schwerer Teig in Google.at nach und findet diesen } \\
\text { Ausdruck dort in einem entsprechenden Kontext, was ihn } \\
\text { dazu veranlasst, diesen Ausdruck zu verwenden (+) }\end{array}$ \\
\hline KEG & $\begin{array}{l}\text { 2: Pfannkuchenteig, } \\
\text { Schlagsahne, } \\
\text { Majonäse, Saucen } \\
\text { 3: Eischnee, } \\
\text { Kuchenteig, } \\
\text { Süßspeisen, fester } \\
\text { Teig [sic] } \\
\text { (akzeptabel) }\end{array}$ & $\begin{array}{l}\text { keine } \\
\text { keine Erwähnung im } \\
\text { versuchsbegleitenden } \\
\text { Fragebogen }\end{array}$ & $\begin{array}{l}\text { P4 (de-DE oder de-AT) - keine Nachschlagevorgänge } \\
\text { Vpn entscheidet sich für bundesdeutsche Benennungen } \\
\text { aufgrund der zahlenmäßigen Überlegenheit der } \\
\text { Bundesdeutschen (+) } \\
\text { übersetzt die einzelnen Mixgüter ohne Nachschlagen Wort } \\
\text { für Wort ohne Reflexion generell zu erfüllender Kriterien, } \\
\text { was zu einer akzeptablen Lösung führt und daher positiv zu } \\
\text { bewerten ist (+) - negativ zu bewerten wäre dies gewesen, } \\
\text { wenn er zur wörtlichen Übersetzung aufgrund von } \\
\text { Rezeptionsproblemen hätte nachschlagen müssen, was } \\
\text { ineffizient gewesen wäre aufgrund der Möglichkeit, einfach } \\
\text { typische (auch andere) Beispiele anzuführen }\end{array}$ \\
\hline
\end{tabular}

11 Idealerweise erkennt der Übersetzer bei Problemstelle 3, dass es nicht nötig ist, im Zieltext Denotatsgleichheit herzustellen, und erspart sich das Nachschlagen bei ihm unbekannten Ausdrücken, so dass hier streng genommen jeder Nachschlagevorgang negativ zu werten wäre. Andererseits muss man erst einmal wissen, um welche Beispiele es sich im AT handelt, um daraus schließen zu können, dass es um dünnflüssige bzw. dickflüssige Mixgüter geht, so dass Nachschlagevorgänge zur Lösung von Verstehensproblemen bei Beispiel 3 im Gegensatz zu solchen zur Lösung von Wiedergabeproblemen nicht negativ gewertet werden.

12 Gebäck müsste ggf. auch als fehlerhaft bewertet werden, da nicht das Gebäck selbst gerührt wird, sondern der Gebäckteig. Gebäck dürfte in diesem Kontext aber richtig verstanden werden, weswegen es nicht als fehlerhaft gewertet wurde. Zu beachten ist, dass mit der Bezeichnung Gebäck in Deutschland und in Österreich unterschiedliche Begriffe verknüpft werden. In Deutschland versteht man unter Gebäck süßes Feingebäck, wie z. B. Weihnachtsplätzchen, in Österreich hingegen ist Gebäck der „Oberbegriff für Semmel, Weckerl usw.“ (Duden 1998). 


\begin{tabular}{|c|c|c|c|}
\hline LEB & $\begin{array}{l}\text { 2: Omelettenteig } \\
\text { [sic], } \\
\text { Schlagsahne, } \\
\text { Mayonnaise, } \\
\text { Saucen } \\
\text { 3: Eischnee, } \\
\text { Kuchen, Pasteten } \\
\text { [sic], Brotteig } \\
\text { (nicht akzeptabel, } \\
\text { weil Pasteten } \\
\text { keine typischen } \\
\text { Beispiele für } \\
\text { Rührgut sind, } \\
\text { sondern eher } \\
\text { gekauft als selbst } \\
\text { hergestellt } \\
\text { werden) }\end{array}$ & $\begin{array}{l}\text { Kuchenteig } \\
\text { Kuchenmischung } \\
\text { Mürbeteig } \\
\text { Kuchenteig } \\
\text { feste Teige } \\
\text { cake mix, pastry, heavy } \\
\text { dough - „Was sind die } \\
\text { Unterschiede?“-Vpn ist } \\
\text { mit ihren Lösungen } \\
\text { „gänzlich unzufrieden“. }\end{array}$ & $\begin{array}{l}\text { P4 (cake mix), P5 (pastry), P6 (heavy dough) - } 3 \\
\text { Nachschlagevorgänge } \\
\text { Vpn ist sich der Bedeutung von cake mix, pastry und heavy } \\
\text { dough nicht sicher, schließt aber, dass es sich jeweils um } \\
\text { Teige handeln muss (+) } \\
\text { übersetzt cake mix spontan mit Kuchenmischung (-) } \\
\text { schlägt pastry, dough und heavy dough im zweisprachigen } \\
\text { Oxford-Duden nach und ist mit den Resultaten unzufrieden, } \\
\text { da es sich in allen Fällen um doughs handelt (+) } \\
\text { spätestens diese Erkenntnis müsste sie dazu bewegen, einfach } \\
\text { Beispiele für zähflüssigere Mixgüter zu finden, was aber } \\
\text { nicht geschieht, sie schreibt etwas hin, was sie nicht } \\
\text { zufrieden stellt (-) }\end{array}$ \\
\hline RAN & $\begin{array}{l}\text { 2: Pfannkuchen- } \\
\text { teig, Schlagsahne, } \\
\text { Mayonnaise, } \\
\text { Saucen } \\
\text { Eischnee, Kuchen } \\
\text { und Torten [sic], } \\
\text { schwere } \\
\text { Teigsorten } \\
\text { (nicht akzeptabel, } \\
\text { weil nicht die } \\
\text { Kuchen und } \\
\text { Torten an sich } \\
\text { gerührt werden, } \\
\text { sondern die Teige } \\
\text { und Cremes für } \\
\text { sie) }\end{array}$ & $\begin{array}{l}\text { Pfannkuchenteig } \\
\text { Omelettteig } \\
\text { Eischnee } \\
\text { Kuchenteig } \\
\text { schwerer Teig } \\
\text { keine Erwähnung im } \\
\text { versuchsbegleitenden } \\
\text { Fragebogen }\end{array}$ & $\begin{array}{l}\text { P4 (pastry) - } 1 \text { Nachschlagevorgang } \\
\text { erkennt das Problem der unterschiedlichen Benennungen in } \\
\text { den deutschsprachigen Ländern und entscheidet sich für die } \\
\text { bundesdeutschen Varianten (+) } \\
\text { schlägt pastry im zweisprachigen Wörterbuch Leo nach (+) } \\
\text { und entscheidet sich dann für Kuchen und Torten } \\
(-) \text { [könnte aber strategisches Vorgehen sein, Problem } \\
\text { besteht nur in der in der ersten Spalte in der Klammer } \\
\text { angegebenen Begründung] }\end{array}$ \\
\hline
\end{tabular}

Tab. 5. Übersetzungsproblem „Beispiele für Einsatz der Geschwindigkeitsstufen“ (prof. Übersetzer)

\begin{tabular}{|c|c|c|c|}
\hline Vpn & Version im ZT & $\begin{array}{l}\text { in Erwägung gezogene } \\
\text { Alternativen (auch in } \\
\text { Wörterbüchern gefundene } \\
\text { Äquivalente) } \\
\text { Kommentare im } \\
\text { versuchsbegleitenden } \\
\text { Fragebogen }\end{array}$ & Lösungsansatz \\
\hline BKR & $\begin{array}{l}\text { 2: Pfannkuchen- } \\
\text { teig, Schlagsahne, } \\
\text { Mayonnaise, } \\
\text { Saucen } \\
\text { 3: Eiklar, } \\
\text { Backmischungen, } \\
\text { Feingebäck, dicke } \\
\text { Teige [sic] } \\
\text { (akzeptabel) }\end{array}$ & $\begin{array}{l}\text { Palatschinkenteig } \\
\text { Eierkuchenteig } \\
\text { Crêpe } \\
\text { Pasta, } \\
\text { Feingebäck } \\
\text { Pastete } \\
\text { Teig } \\
\text { Hefeteig } \\
\text { schwerer Teig } \\
\text { Sauerteig } \\
\text { heavy dough - Vpn ist mit } \\
\text { ihrer Lösung } \\
\text { unberechtigterweise „eher } \\
\text { zufrieden“. }\end{array}$ & $\begin{array}{l}\text { P5, P5.2, P5.3 (pancake batter), P6, P6.2, P6.3, P6.4 (pastry), } \\
\text { P7, P7.2, P7.3, P7.4 (heavy dough/mixture), P20, P20.2 } \\
\text { (Palatschinken/Pfannkuchen), P21, P21.2, P21.3 (whipped } \\
\text { cream), P24 (eggwhite), P26, P26.2 (cake mix) - 7 } \\
\text { Nachschlagevorgänge mit bis zu } 3 \text { Wiederaufnahmen } \\
\text { kennt die Bedeutung von pastry nicht und schlägt in der } \\
\text { Orientierungsphase in Leo nach (+), wo sie Pastete und } \\
\text { Feingebäck findet, was sie kommentiert mit: ,jetz muss i } \\
\text { wissn, was von den zeig des is“ (-) } \\
\text { schlägt heavy dough ebenfalls in der Orientierungsphase in Leo } \\
\text { nach und findet die Kollokation nicht, zieht dicker, schwerer } \\
\text { und schwieriger Teig in Erwägung (+) } \\
\text { kennt die Bedeutung von pancake batter nicht und schlägt in } \\
\text { Wiktionary nach, wo sie anhand der englischen Erklärung das } \\
\text { Verstehensproblem lösen kann (+) (Vpn schaut später auch } \\
\text { nach, ob dort eine dt. Entsprechung angegeben wird, was aber } \\
\text { nicht der Fall ist (-)) } \\
\text { erkennt die Problematik der Kulturspezifik des engl. Ausdrucks } \\
\text { (+) und das Problem der unterschiedlichen Benennungen in den } \\
\text { deutschsprachigen Ländern und entscheidet sich am Ende für } \\
\text { die bundesdeutschen Varianten, obwohl sie zunächst } \\
\text { Palatschinkenteig schreibt, bei Schlagsahne/Schlagobers } \\
\text { temporär beide Varianten angibt und sich negativ gegenüber } \\
\text { dem Bundesdeutschen äußert: ,i schreib net so blöd } \\
\text { Hochdeutsch Deutschland-Deutsch“ (+) }\end{array}$ \\
\hline
\end{tabular}




\begin{tabular}{|c|c|c|c|}
\hline & & & $\begin{array}{l}\text { schlägt auch Mayonnaise und mayonnaise in der dt. und engl. } \\
\text { Wikipedia nach, um festzustellen, ob man darunter auch } \\
\text { dasselbe versteht (-) [überflüssiger Nachschlagevorgang } \\
\text { insofern, als es nicht auf Denotatsgleichheit ankommt, zeigt } \\
\text { aber Sensibilität für Interferenzvermeidung] } \\
\text { assoziiert zu egg whites sofort Eischnee, ist sich aber nicht } \\
\text { sicher, ob dies die übliche dt. Bezeichnung ist, und schlägt } \\
\text { umfassend und sehr lange zum Thema „Ei“ nach, wobei sie } \\
\text { auch Eischnee findet, aber schließlich Eiklar verwendet (-) } \\
\text { startet lange Nachschlageketten zu pastry, das sie zunächst mit } \\
\text { Pasta assoziiert, und verliert sich beim Nachschlagen auch im } \\
\text { weiteren Nachschlagen von ihr unbekannten Wörtern, die in } \\
\text { Erklärungen vorkommen (Vpn verfolgt hier eindeutig nicht das } \\
\text { Ziel, die Übersetzung rasch abzuschließen, sondern sich dabei } \\
\text { umfassend weiterzubilden) (-); am Ende trifft sie aber die } \\
\text { korrekte Entscheidung für Feingebäck (+) } \\
\text { sucht heavy dough in Google, schlägt heavy in Leo nach, } \\
\text { assoziiert Hefeteig und schlägt in Leo nach, was Hefe auf } \\
\text { Englisch heißt, um auszuschließen, dass mit heavy dough } \\
\text {,Hefeteig’ gemeint ist (-) } \\
\text { schlägt cake und cake mix in Leo nach und in der } \\
\text { Revisionsphase auch noch in zahlreichen anderen Nach- } \\
\text { schlagewerken, um herauszufinden, ob damit Kuchenteig oder } \\
\text { Backmischung gemeint ist (-), Vpn entscheidet sich dann } \\
\text { korrekterweise für Backmischungen (+) } \\
\text { in der Revisionsphase folgen außerdem lange Nach- } \\
\text { schlageketten zu pastry und heavy dough (-) }\end{array}$ \\
\hline KNI & $\begin{array}{l}\text { 2: Palatschinken- } \\
\text { teig, Schlagobers, } \\
\text { Mayonnaise, } \\
\text { Saucen } \\
\text { 3: Eischnee, } \\
\text { Kuchen- und } \\
\text { Gebäcksteig [sic], } \\
\text { dicke Teige [sic] } \\
\text { (akzeptabel) }\end{array}$ & $\begin{array}{l}\text { Eierkuchenteig } \\
\text { Omelettteig } \\
\text { Pastete } \\
\text { Gebäck } \\
\text { Feingebäck } \\
\text { dicker Teig } \\
\text { schwere Teige } \\
\text { plumpe Teige } \\
\text { Hefeteig } \\
\text { heavy dough/mixture - Vpn } \\
\text { ist mit ihrer Lösung „eher } \\
\text { zufrieden“. }\end{array}$ & $\begin{array}{l}\text { P2, P2.2, P2.3, P2.4 (heavy dough), P16, P16.2 (pancake } \\
\text { batter), P17 (whipped cream), P18 (egg whites), P19, P19.2 } \\
\text { (cake mix), P20 (pastry) - } 6 \text { Nachschlagevorgänge mit bis zu } 3 \\
\text { Wiederaufnahmen } \\
\text { schlägt pancake und batter in Leo nach und löst damit ihr } \\
\text { Verstehensproblem (+) } \\
\text { erkennt das Problem der unterschiedlichen Benennungen in den } \\
\text { deutschsprachigen Ländern, sucht nach „neutralen“ } \\
\text { Bezeichnungen, was ihr nicht zu gelingen scheint, und } \\
\text { entscheidet sich dann konsistent für die österreichischen } \\
\text { Bezeichnungen (+) } \\
\text { schlägt whipped cream nur deshalb in Leo nach, um die } \\
\text { bundesdeutsche Bezeichnung dafür herauszufinden, und findet } \\
\text { auch Schlagsahne (+) } \\
\text { Vpn entscheidet sich für österreichische Bezeichnungen, weil } \\
\text { sie die dt. (zumindest z. T.) noch nie wo gelesen hat und daher } \\
\text { nicht verwenden möchte (+) } \\
\text { schlägt egg white in Leo nach, weil sie sich der üblichen dt. } \\
\text { Bezeichnung nicht sicher ist, und entscheidet sich dann für } \\
\text { Eischnee (+) } \\
\text { assoziiert mit cake mix spontan Backmischungen, ist sich aber } \\
\text { nicht sicher und schlägt in Leo nach, wo sie keinen Eintrag } \\
\text { findet, und bleibt dann zunächst bei Backmischungen (+) } \\
\text { schlägt pastry, dough, heavy und heavy dough in Leo nach (+) } \\
\text { Backmischungen und Gebäcksteig gefällt der Vpn weniger als } \\
\text { Kuchen- und Gebäcksteig, für das sie sich daher entscheidet (+) }\end{array}$ \\
\hline SFR & $\begin{array}{l}\text { 2: Pfannkuchen- } \\
\text { teig, Schlagsahne, } \\
\text { Mayonnaise, } \\
\text { Soßen } \\
\text { 3: Eiweiß, } \\
\text { Kuchenteig, } \\
\text { Gebäck, schwerer } \\
\text { Teig } \\
\text { (akzeptabel) }\end{array}$ & $\begin{array}{l}\text { Kuchenmischung } \\
\text { keine Erwähnung im } \\
\text { versuchsbegleitenden } \\
\text { Fragebogen }\end{array}$ & $\begin{array}{l}\text { P15 (batter), P16 (whipped cream), P18 (cake mix), P19 } \\
\text { (pastry), P20, P20.2 (heavy dough) - } 5 \text { Nachschlagevorgänge } \\
\text { mit } 1 \text { Wiederaufnahme } \\
\text { schlägt batter in Leo nach und verwendet danach die korrekte } \\
\text { Übersetzung Pfannkuchenteig (+) } \\
\text { schlägt whipped cream im zweisprachigen Online-Wörterbuch } \\
\text { Leo nach, obwohl sie zumindest ahnt, dass es Sahne heißt (-) } \\
\text { assoziiert cake mix spontan mit Kuchenmischung, ist sich } \\
\text { unsicher und schlägt es in Leo nach (+), wonach sie es mit } \\
\text { Kuchenteig übersetzt (+) }\end{array}$ \\
\hline
\end{tabular}




\begin{tabular}{|c|c|c|c|}
\hline & & & $\begin{array}{l}\text { assoziiert mit pastry spontan Gebäck, schlägt aber dennoch in } \\
\text { Leo nach, wodurch sie sich bestätigt fühlt (-) } \\
\text { assoziiert mit heavy dough sofort schwerer Teig, schlägt aber } \\
\text { dennoch in Leo nach, weil sie vermutet, es könnte „etwas } \\
\text { Bestimmtes“ heißen, was sich nicht bestätigt (+) }\end{array}$ \\
\hline HHE & $\begin{array}{l}\text { 2: Pfannkuchen- } \\
\text { teig, Schlagsahne, } \\
\text { Mayonnaise, } \\
\text { Soßen } \\
\text { 3: Eiweiß, } \\
\text { Kuchenteig, Teig, } \\
\text { schweren Teig } \\
\text { [sic] } \\
\text { (nicht akzeptabel) }\end{array}$ & $\begin{array}{l}\text { keine Erwähnung im } \\
\text { versuchsbegleitenden } \\
\text { Fragebogen }\end{array}$ & $\begin{array}{l}\text { P17 (batter), P18 (pancake), P19 (whipped cream), P21 (egg } \\
\text { white), P22 (cake mix), P23 (pastry), P24 (dough) - } 7 \\
\text { Nachschlagevorgänge } \\
\text { kennt die Bedeutung von batter, pancake und whipped nicht, } \\
\text { schlägt diese Wörter in Leo nach und verwendet dann korrekt } \\
\text { Pfannkuchenteig und Schlagsahne (+) } \\
\text { überlegt, wie sie egg whites am besten übersetzt, schlägt es in } \\
\text { Leo nach, findet Eiweiß und Eischnee und verwendet ersteres, } \\
\text { was akzeptabel ist, aber nicht ideal (-) } \\
\text { schlägt cake mix, obwohl sie dazu Teigmischungen assoziiert } \\
(+), \text { pastry und dough in Leo nach und verwendet dann die } \\
\text { Version im ZT, obwohl sie dazu anmerkt „es is wieder } \\
\text { dasselbe“ (-) }\end{array}$ \\
\hline TDI & $\begin{array}{l}\text { 2: Pfannkuchen- } \\
\text { teig, Schlagsahne, } \\
\text { Mayonaise [sic], } \\
\text { Soßen } \\
\text { 3: Eischnee, } \\
\text { Kuchen- } \\
\text { mischungen, } \\
\text { Gebäck, schwere } \\
\text { Teigmasse } \\
\text { (akzeptabel) }\end{array}$ & $\begin{array}{l}\text { fester Teig } \\
\text { schwerer Teig } \\
\text { schwere Teigmasse } \\
\text { Pasta } \\
\text { heavy dough - Vpn ist mit } \\
\text { seiner Lösung „eher } \\
\text { zufrieden“. }\end{array}$ & $\begin{array}{l}\text { P10 (batter), P11 (egg white), P12 (cake mix), P13 (dough), } \\
\text { P27 (pastry) - } 5 \text { Nachschlagevorgänge } \\
\text { schlägt batter in Leo nach und verwendet danach die korrekte } \\
\text { Übersetzung Pfannkuchenteig (+) } \\
\text { assoziiert zu egg whites spontan Eiweiß(e), fragt sich aber, wie } \\
\text { man das üblicherweise im Deutschen nennt, und schlägt in Leo } \\
\text { nach, wo sie auch Eischnee findet, das ihm plausibler erscheint, } \\
\text { weil man für Eiweiß keinen Mixer brauche (+) } \\
\text { kennt die Bedeutung von cake mix nicht und schlägt erfolgreich } \\
\text { in Leo nach (+) } \\
\text { übersetzt pastry spontan mit Pasta (keine Denotatsgleichheit, } \\
\text { aber sinnvolles Beispiel, das aber nicht auf strategischem Wege } \\
\text { gefunden wurde) (-) } \\
\text { kennt die Bedeutung von dough nicht und schlägt erfolgreich in } \\
\text { Leo nach (+) } \\
\text { fragt sich in der Revisionsphase nach dem Plural von Pasta, } \\
\text { erwägt Übersetzungsalternativen (vielleicht } \\
\text { Problemvermeidungsstrategie) und schlägt Mehrzahl Pasta in } \\
\text { Google nach, dann pastry in Leo, wo sie u.a. auch auf Pastete } \\
\text { und Gebäck stößt, verwendet Gebäck (+) }\end{array}$ \\
\hline $\mathrm{KBE}$ & $\begin{array}{l}\text { 2: Pfannkuchen- } \\
\text { teig, Schlagsahne, } \\
\text { Mayonnaise, } \\
\text { Saucen } \\
\text { 3: Eischnee, } \\
\text { Kuchengemisch } \\
\text { [sic], Gebäck, } \\
\text { schwerer Teig } \\
\text { (nicht akzeptabel, } \\
\text { weil } \\
\text { Kuchengemisch } \\
\text { nicht verständlich } \\
\text { ist) }\end{array}$ & $\begin{array}{l}\text { Kuchenteig } \\
\text { Kuchengemisch } \\
\text { keine Erwähnung im } \\
\text { versuchsbegleitenden } \\
\text { Fragebogen }\end{array}$ & $\begin{array}{l}\text { P15 (pancake batter), P16 (whipped cream), P17 (egg whites), } \\
\text { P18 (cake mix), P19; P19.2 (pastry), P20 (heavy dough) - } 6 \\
\text { Nachschlagevorgänge } \\
\text { schlägt pancake, batter, whipped cream und egg whites im } \\
\text { zweisprachigen Online-Wörterbuch dict.cc nach (-) und } \\
\text { verwendet danach korrekte Übersetzungen (+) } \\
\text { schlägt cake mix, mix, pastry und dough im zweisprachigen } \\
\text { Online-Wörterbuch dict.cc nach und überlegt, ob sie cake mix } \\
\text { mit Kuchenteig oder Kuchenmischung übersetzen soll (-) } \\
\text { scheint pastry auch als Teig zu verstehen, was sie veranlasst, } \\
\text { Kuchenteig in Kuchenmischung abzuwandeln (was wenig } \\
\text { sinnvoll ist, da die Mischung ja auch einen Teig ergibt) (-) } \\
\text { ist sich unsicher, ob sie pastry korrekt verstanden hat und } \\
\text { schlägt es mit dem Kommentar ,kann ja net zwamol des } \\
\text { Gleiche sein“ erneut im selben Wörterbuch nach (-) }\end{array}$ \\
\hline
\end{tabular}

Tab. 6. Übersetzungsproblem „Beispiele für Einsatz der Geschwindigkeitsstufen“ (Novizen)

Betrachten wir nun noch eine Auffälligkeit: BKR schlägt extrem viel nach und verfolgt lange Nachschlageketten, in denen sie sich durch Ausdrücke, die sie in Erklärungen findet, aber nicht kennt, wieder dazu verleiten lässt, diese nachzuschlagen. Dieses Verhalten ist unter professionellen Gesichtspunkten ineffizient, es ist jedoch - angesichts der Tatsache, dass BKR eine Novizin im ersten Semester ist, - eine gute Strategie, ihre Sprachkompetenz auszubauen. Ihre Lösungsansätze, die oftmals mit denjenigen der professionellen Übersetzer konkurrieren können, und ihre Tendenz, sich erst mit einer Lösung zufrieden zu geben, wenn sie sich deren Korrektheit 
relativ sicher ist, sowie ihr kritisches Hinterfragen der Sinnhaftigkeit des AT zeugen von hoher Motivation und einem hohem Potenzial dafür, dass sie eine gute Übersetzerin wird.

\section{6. $\quad$ Fazit}

Die Analysen zeigen, dass professionelle Übersetzer effizienter arbeiten als Novizen, stärker strategiegeleitet vorgehen, mehr Reflexionen auf makrostrategischer Ebene anstellen und dabei insgesamt - wenn auch nicht an allen hier im Detail untersuchten Textstellen - weniger Fehler machen, was auch nicht anders zu erwarten war. Was aber erstaunt, ist, dass auch die professionellen Vpn noch weit von dem entfernt sind, was man von einem Übersetzungsexperten hätte erwarten können. Das mag daran liegen, dass die Textsorte Anleitung nicht zu denjenigen Textsorten gehören mag, mit denen die hier untersuchten professionellen Übersetzer in ihrem Berufsalltag üblicherweise konfrontiert werden. Die professionelle Übersetzerin RAN gibt beispielsweise an, eine Bedienungsanleitung zuletzt vor zehn Jahren übersetzt zu haben. Die Probleme, die in diesem Aufsatz näher betrachtet wurden, sind jedoch keine bedienungsanleitungsspezifischen Probleme: terminologische Probleme wie im Falle des control switch, missverständliche Aussagen im AT wie im Falle des while you are using it und kulturspezifische Aufzählungen, bei denen es nicht auf Denotatsgleichheit ankommt, können in jeder Textsorte vorkommen. Die hier aufgezeigten idealen Lösungsstrategien sollten zum Repertoire jedes Berufsübersetzers gehören, gleich auf welche Textsorten er oder sie spezialisiert ist. Das gilt auch für literarische Übersetzer, denn beim literarischen Übersetzen stellen sich solche Probleme auch. Studierende für die Kriterien, die die jeweiligen Übersetzungen erfüllen müssen, zu sensibilisieren und ihnen ideale Lösungswege aufzuzeigen dürfte sich in der Übersetzungsdidaktik bewähren. Das gilt aber nicht nur für Studierende, sondern, wie die Ergebnisse der hier untersuchten Berufsübersetzer zeigen, sicher auch für professionelle Übersetzer, denen entsprechende Weiterbildungsangebote gemacht werden sollten. Und nicht zuletzt dürften die Probleme, die ihre Texte Übersetzern machen, auch für Technische Redakteure aufschlussreich sein (die oftmals deren Urheber sind), wenn es darum geht, ihre Textproduktionskompetenz zu verbessern.

Wie bei den Fehleranalysen deutlich wurde, zeigen sich die größten Schwächen bei beiden Gruppen von Vpn in der Semantik. Dies ist ein Hinweis darauf, dass die muttersprachliche Kompetenz unserer Studierenden verbessert werden muss. Haben selbst Studierende in einem kommunikationsorientierten Studiengang wie dem Übersetzern in diesem Bereich zu Beginn ihres Studiums und, wie die professionellen Übersetzer zeigen, auch später Schwierigkeiten, so kann dies wohl von den meisten Studienanfängern, insbesondere auch solchen in weniger kommunikationsorientierten Studiengängen, angenommen werden. Um festzustellen, ob Studierende in der Lage sind, feine semantische Unterschiede bei der Rezeption von Texten zu erkennen und sie auch beim Produzieren von Texten zum Ausdruck zu bringen, sind Übersetzungen ideale Übungen. Denn hier kann im Gegensatz zur freien Textproduktion exakt festgelegt werden, was zum Ausdruck gebracht werden soll, ohne dabei schon die erforderlichen sprachlichen Mittel vorzugeben. Insofern betrachte ich Übersetzungen zwischen dem Englischen als internationaler lingua franca (aber auch einer anderen von den Studierenden auf hohem Niveau beherrschten Fremdsprache) und der Muttersprache als eine Möglichkeit zur Verbesserung der Textrezeptions- und Textproduktionskompetenz.

\section{Bibliographie}

Anderson, John R. ${ }^{6} 2007$ : Kognitive Psychologie. Berlin, Heidelberg: Springer.

Chesterman, Andrew 1997: Memes of Translation: The Spread of Ideas in Translation Theory. Amsterdam, Philadelphia: Benjamins.

Dreyfus, Hubert L./Dreyfus, Stuart E. 1986: Mind over Machine. Oxford: Blackwell.

Duden 1998: Wie sagt man in Österreich? Wörterbuch des österreichischen Deutsch. 3. Aufl. Mannheim, Leipzig, Wien, Zürich: Dudenverlag. 
Ehrensberger-Dow 2009: Projekt „Capturing Translation Processes“ an der Zürcher Hochschule für Angewandte Wissenschaften [online]. http://www.zhaw.ch/fileadmin/php_includes/popup/projekt-detail.php?projektnr=395 (accessed 10 August 2009).

Ernst, Richard: Wörterbuch der industriellen Technik. Wiesbaden: Brandstätter.

Ericsson, K. Anders/Simon, Herbert A. 1993 [1984]: Protocol Analysis: Verbal Reports as Data. Rev. Ed. Cambridge (Mass.), London (England): MIT Press.

Ericsson, K. Anders/Smith, Jacqui 1991: Prospects and limits of the empirical study of expertise: An introduction. In Ericsson, K. Anders/Smith, Jacqui (Hrsg.) Towards a General Theory of Expertise. Prospects and Limits. Cambridge: Cambridge Univ. Press, 1-38.

Gerloff, Pamela 1988: From French to English: A look at the translation process in students, bilinguals, and professional translatorss. (Unveröffentlichte Dissertation). Cambridge (MA): Harvard University.

Göpferich, Susanne 1995: Textsorten in Naturwissenschaften und Technik: Pragmatische Typologie - Konstrastierung - Translation. Tübingen: Narr.

Göpferich, Susanne 2008: Translationsprozessforschung: Stand - Methoden - Perspektiven. Tübingen: Narr.

Göpferich, Susanne 2009a: Towards a model of translation competence and its acquisition: the longitudinal study 'TransComp'. In Göpferich, Susanne/Jakobsen, Arnt Lykke/Mees, Inger M. (Hrsg.) Behind the mind: Methods, models and results in translation process research. (Copenhagen Studies in Language 37). Copenhagen: Samfundslitteratur Press, 11-37.

Göpferich, Susanne 2009b: Datendokumentation zum Artikel “Anleitungen rezipieren, Anleitungen produzieren: Empirische Befunde zu kognitiven Prozessen bei Übersetzungsnovizen und Übersetzungsprofis [online]. http://www. susanne-goepferich.de/data-documentation-t1-t8-A3-B3.pdf (accessed 10 August 2009).

Göpferich, Susanne 2010: Data documentation and data accessibility in translation process research. In The Translator $16: 1$.

Göpferich, Susanne/Bayer-Hohenwarter, Gerrit/Stigler, Hubert (Hrsg.) 2008 ff.: TransComp - The Development of Translation Competence. Korpus und Asset-Management-System zur Longitudinalstudie TransComp. Graz: KarlFranzens-Universität [online]. http://gams.uni-graz.at/container:tc (accessed 10 August 2009).

Jakobsen, Arnt Lykke 1999: Logging target text production with Translog. In Hansen, Gyxe (Hrsg.) Probing the Process in Translation: Methods and Results. (Copenhagen Studies in Language 24). Copenhagen: Samfundslitteratur, $9-20$.

Jakobsen, Arnt Lykke 2002: Translation drafting by professional translators and by translation students. In Hansen, Gyde (Hrsg.) Empirical Translation Studies: Process and Product. (Copenhagen Studies in Language 27). Copenhagen: Samfundslitteratur, 191-204

Jääskeläinen, Riitta/Tirkkonen-Condit, Sonja 1991: Automised processes in professional vs. non-professional translation: A think-aloud protocol study. In Tirkkonen-Condit, Sonja (Hrsg.) Empirical Research in Translation and Intercultural Studies: Selected Papers of the TRANSIF Seminar, Savonlinna 1988. Tübingen: Narr, 89-109.

Künzli, Alexander 2003: Quelques stratégies et principes en traduction technique français-allemand et français-suédois. (Forskningsrapporter Cahiers de la recheche 21). Stockholm: Stockholms universitet, Institutionen för franska och italienska. 


\section{Anhang A: Ausgangstext mit Übersetzungsauftrag}

\section{Übersetzungsauftrag:}

Dieser Text stammt aus der Bedienungsanleitung für den Moulinex-Handmixer mit der Produktbezeichnung SUPERMIX 180 (A28). Die Abbildung 2 unten kann herausgeklappt werden, so dass man sie parallel zum Text betrachten kann.

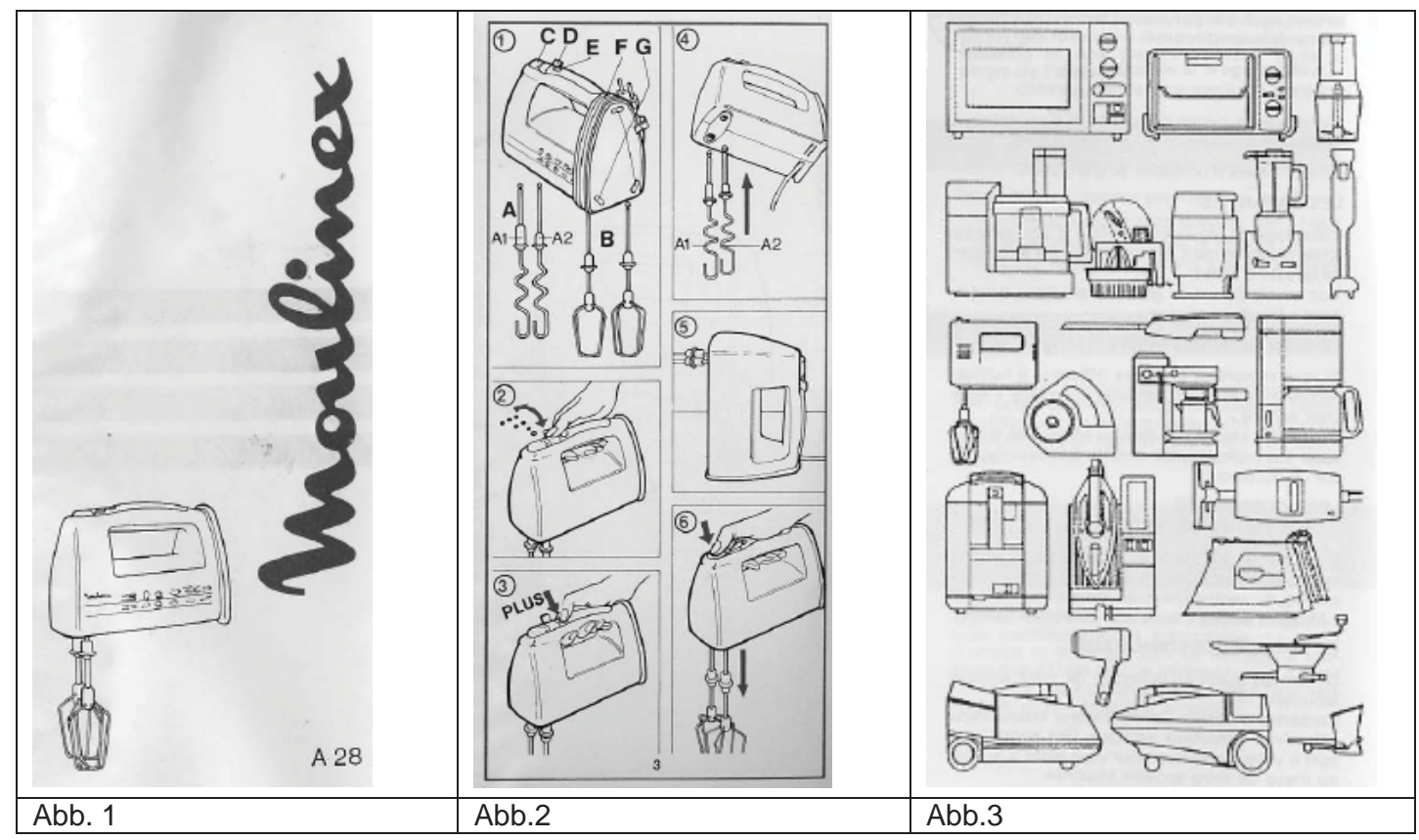

Nach einer kurzen Einleitung werden die folgenden Instruktionen [nicht zu übersetzen!] gegeben, denen dann der von Ihnen zu übersetzende Text folgt:

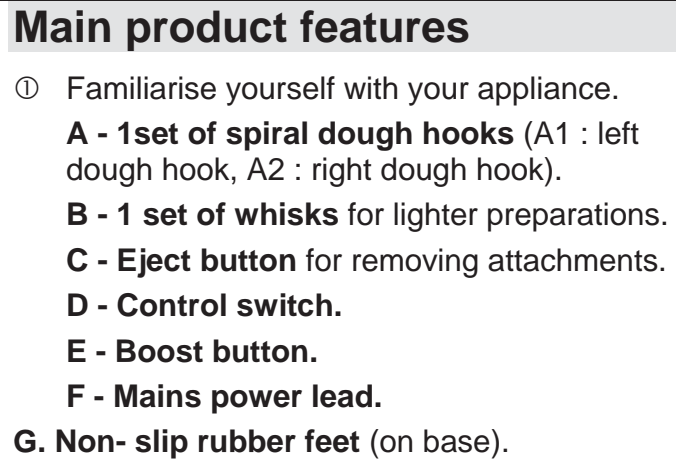

(1) Familiarise yourself with your appliance.

A - 1set of spiral dough hooks (A1 : left dough hook, A2 : right dough hook).

B - 1 set of whisks for lighter preparations.

C - Eject button for removing attachments.

D - Control switch.

E - Boost button.

F - Mains power lead.

G. Non- slip rubber feet (on base).

\section{Technical information}

(2) Speeds selected with control switch (D) :

$$
1 \text { : Slow } 2 \text { : Medium } 3 \text { : Fast }
$$

(3) The "boost" button (E)gives an instantaneous, high speed boost with more power for a better finish to mixtures.

NB: It can be used with any of the 3 speeds.

\section{Safety instructions}

\section{[...] [nicht zu übersetzen!]}

Bitte übersetzen Sie den nachfolgenden Text für die deutschsprachigen Benutzer. Die deutsche Version soll in derselben (mehrsprachigen) Broschüre erscheinen. 


\section{Moulinex Supermix}

\section{Using your mixer}

(4) Check that control switch $\mathrm{D}$ is in position 0 , then insert the attachments (ie. Dough hook A as indicated).

Select the speed (see recommendations).

(5) You can stand the appliance on its base while you are using it.

(6) Removal of attachments. With the control switch (D) on 0, the attachments can be removed simply by pressing the eject button.

\section{Recommendations}

Slow speed (1) is used to start any mixing or whisking (it avoids splashing).

Medium and fast speeds are used depending on the job to be done.

Examples:

- Medium speed (2): pancake batter, whipped cream, mayonnaise, sauces.

- Fast speed (3) egg whites, cake mix, pastry, heavy dough.
(5) - Boost button : to incorporate ingredients more quickly or to give more power with heavy mixtures when the mixture thickens. You can use the boost button with any speed.

NOTE : Never let the mixture rise above the guard on the attachments. Where there is no guard, never let the mixture rise up the stem of the attachment and into the body of the product.

\section{Cleaning}

- Only the attachments can be washed.

- Never immerse the body of your appliance in water.

- Never run it under the tap.

- Wipe it with a dry or slightly damp cloth.

\section{Important}

If the power supply lead is damaged, it must be replaced by an approved Moulinex Service Agent. Failure to do so invalidates the guarantee.

\section{Anhang B: Fehlerbewertungsschema}

\begin{tabular}{|l|l|}
\hline \hline Fehlerkategorie & Beschreibung/Beispiel \\
\hline \hline Formale Fehler & \\
\hline \hline Zeichensetzung & Zeichensetzungsfehler, meist fehlende oder falsche Kommata \\
\hline Orthographie & $\begin{array}{l}\text { Rechtschreibfehler, bei denen es sich nicht um offensichtliche Tippfehler handelt (z. B. Tauchen } \\
\text { Sie das Gehäuse ihres Gerätes nie unter Wasser.) }\end{array}$ \\
\hline Absatz & $\begin{array}{l}\text { unmotivierte Absatzbildung (die Vpn hatten programmbedingt nicht die Aufgabe, weitere } \\
\text { Formatierungen im Text vorzunehmen) }\end{array}$ \\
\hline \hline Wortwahlfehler & \multicolumn{2}{|l|}{} \\
\hline \hline Semantik & $\begin{array}{l}\text { Bedeutungs-/Sinnveränderung auf denotativer oder konnotativer Ebene; Verwendung eines nicht } \\
\text { existierenden Ausdrucks; Auslassung relevanter Informationen; falsches Register auf der } \\
\text { Wortebene }\end{array}$ \\
\hline Kollokation & Kollokationsfehler (z. B. schnelle Geschwindigkeit statt hohe Geschwindigkeit) \\
\hline Präposition & $\begin{array}{l}\text { auf eine Kontamination zurückgehender Fehler (Verschmelzung zweier sprachlicher Einheiten } \\
\text { oder Konstruktionen, die zeitgleich im Arbeitsgedächtnis wachgerufen werden) }\end{array}$ \\
\hline \hline
\end{tabular}

13 Streng genommen ist die Verwendung von Präpositionalphrasen anstellen eines Genitivs als grammatischer Fehler zu werten; da diese Fehlerkategorie aber eng mit der Präpositionsverwendung verknüpft ist, wurde der Einfachheit halber auf eine Aufgliederung der Präpositionsfehler in eine lexikalische und eine grammatische Subkategorie verzichtet. 


\begin{tabular}{|c|c|}
\hline \multicolumn{2}{|l|}{ Grammatikfehler } \\
\hline Tempus & Tempusfehler \\
\hline $\begin{array}{l}\text { Kasus, Numerus, } \\
\text { Kongruenz }\end{array}$ & $\begin{array}{l}\text { Kasus-, Numerus- und Kongruenzfehler (Kasusfehler meist nach Präpositionen oder in } \\
\text { Appositionen) }\end{array}$ \\
\hline Modus & Modusfehler, z. B. in indirekter Rede \\
\hline Syntax & $\begin{array}{l}\text { Syntaxfehler (z. B. falscher Bezug einer Negation, aufgrund von Schachtelung schwer lesbare } \\
\text { Konstruktion) }\end{array}$ \\
\hline Artikel & $\begin{array}{l}\text { falscher Artikelgebrauch (z. B. bestimmter Artikel an Stellen, an denen kein Artikel stehen darf, } \\
\text { oder Verwendung eines bestimmten anstelle eines unbestimmten Artikels) }\end{array}$ \\
\hline $\begin{array}{l}\text { Modalität, } \\
\text { Illokution }^{14}\end{array}$ & falscher Illokutionsindikator, z. B. sollte (Empfehlung) anstelle von muss (Anweisung) \\
\hline Infinitiv & $\begin{array}{l}\text { grammatisch falscher Anschluss einer Infinitivkonstruktion (z. B. Das Wetter war zu schlecht, um } \\
\text { schwimmen zu gehen.) }\end{array}$ \\
\hline \multicolumn{2}{|c|}{ Fehler auf der Textebene } \\
\hline $\begin{array}{l}\text { Teko } \\
\text { (Textkohärenz) }\end{array}$ & $\begin{array}{l}\text { mangelnde Textkohärenz, z. B. fehlende Verbalisierung logisch-semantischer Beziehungen oder } \\
\text { Herstellung falscher logisch-semantischer Beziehungen zwischen Sätzen; falsche/unklare } \\
\text { Proformen; Fehler bei mehrgliedrigen Konjunktionen }\end{array}$ \\
\hline $\begin{array}{l}\text { FSP (funktionale } \\
\text { Satzperspektive) }\end{array}$ & Fehler im Bereich der funktionalen Satzperspektive, wie falsche Thema-Rhema-Strukturen \\
\hline Rhetorik & $\begin{array}{l}\text { Verluste kommunikativen Nachdrucks außer FSP-Fehler (z. B. durch den Ersatz eines Gedichts } \\
\text { durch eine inhaltliche Umschreibung) }\end{array}$ \\
\hline \multicolumn{2}{|l|}{ Sonstige } \\
\hline $\begin{array}{l}\text { Idiomatik/TSK } \\
\text { (Textsorten- } \\
\text { konventionen) }\end{array}$ & $\begin{array}{l}\text { unidiomatische Ausdrucksweise, die nicht zu einer Bedeutungsveränderung führt, aber den Text } \\
\text { klar als Übersetzung im negativen Sinne enttarnt und/oder schwer verständlich macht; Verstoß } \\
\text { gegen die Textsortenkonventionen (z. B. Das Bild ist kein Zufallstreffer. statt Das Bild ist kein } \\
\text { Schnappschuss. und Anfangend mit Namen als Überschrift) }\end{array}$ \\
\hline Kulturspezifik & fehlende Anpassung an die Zielkultur bzw. fehlende kulturelle Neutralisierung \\
\hline
\end{tabular}

14 Streng genommen könnten diese Fehler auch als Wortwahlfehler (Semantik) klassifiziert werden, weil sie eine Auswirkung auf die Bedeutung haben, aber auch als Fehler auf der Textebene (TSK), weil die zu verwendenden Illokutionsindikatoren durch die Textsorte determiniert sein können (s. Göpferich 1995: 308 ff.). 
